\title{
Locality in GNS Representations of Deformation Quantization
}

\section{Stefan Waldmann*}

\author{
Fakultät für Physik \\ Universität Freiburg \\ Hermann-Herder-Str. 3 \\ 79104 Freiburg i. Br., F. R. G
}

FR-THEP-99/2

March 1999

\begin{abstract}
In the framework of deformation quantization we apply the formal GNS construction to find representations of the deformed algebras in pre-Hilbert spaces over $\mathbb{C}[[\lambda]]$ and establish the notion of local operators in these pre-Hilbert spaces. The commutant within the local operators is used to distinguish 'thermal' from 'pure' representations. The computation of the local commutant is exemplified in various situations leading to the physically reasonable distinction between thermal representations and pure ones. Moreover, an analogue of von Neumann's double commutant theorem is proved in the particular situation of a GNS representation with respect to a KMS functional and for the Schrödinger representation on cotangent bundles. Finally we prove a formal version of the Tomita-Takesaki theorem.
\end{abstract}

${ }^{*}$ Stefan.Waldmann@physik.uni-freiburg.de 


\section{Contents}

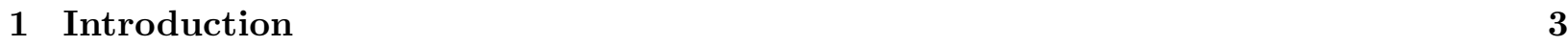

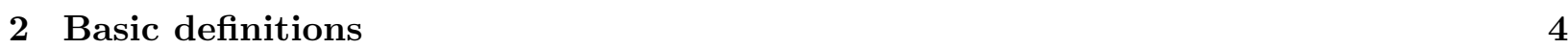

3 Locality in GNS representations and commutants 5

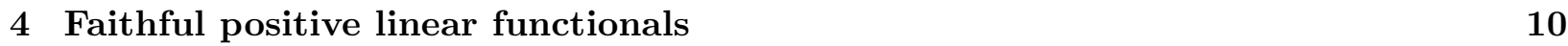

\begin{tabular}{|lr}
\hline 5 Basic examples & 11
\end{tabular}

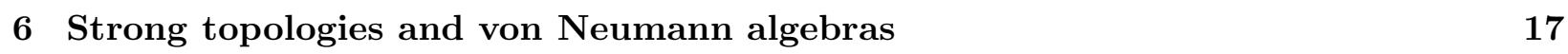

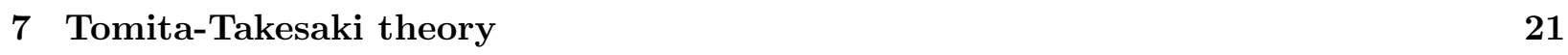

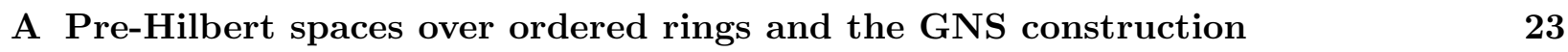

\begin{tabular}{|lr}
\hline B Formal series and $\lambda$-adic topology & 24
\end{tabular} 


\section{Introduction}

The concept of deformation quantization and star products has been introduced by Bayen, Flato, Frønsdal, Lichnerowicz, and Sternheimer in [4] and is now a well-established and successful quantization procedure. The central object of deformation quantization is a star product $*$, a formal associative deformation of the classical Poisson algebra of complex-valued functions $C^{\infty}(M)$ on a symplectic or, more generally, a Poisson manifold $M$, such that in the first order of the formal parameter $\lambda$ the commutator of the star product yields i times the Poisson bracket. Hence $\lambda$ is to be identified with Planck's constant $\hbar$ whenever the formal series converges, and the star product algebra $\left(C^{\infty}(M)[[\lambda]], *\right)$ is viewed as observable algebra of the quantized system corresponding to the classical system described by the Poisson manifold. The existence of such deformations has been shown by DeWilde and Lecomte [16], Fedosov [18, 19], Omori, Maeda, and Yoshioka [28] in the symplectic case and recently by Kontsevich [25] in the general case of a Poisson manifold. Moreover, the star products have been classified up to equivalence by Nest and Tsygan [26, 27], Bertelson, Cahen, and Gutt [5], Fedosov [19], Weinstein and $\mathrm{Xu}$ [36], and Kontsevich [25].

Since the algebra structure is by now quite well-understood the question is raised, how one can encode the notion of states of this algebra. In order to find a physically reasonable notion of states we have introduced in [12] together with Bordemann a formal analogue of the positive functionals and their GNS representation as analogue of the well-known construction from $C^{*}$-algebra theory, see e.g. [14, 15, 20]. In various examples this approach has been successfully applied [6 [10, 12].

While the GNS construction in principle works in a quite general framework (for *-algebras over ordered rings) we shall discuss in this article aspects of these representations which are particular to deformation quantization. Here the observable algebra has an additional structure since the star products are local in the sense that any bilinear operator of the formal star product series is a local or even bidifferential operator. This leads to a 'net structure' of the observable algebra similar to the net structure of the observable algebras in algebraic quantum field theory [20]. It turns out that the spaces $C_{0}^{\infty}(O)[[\lambda]]$ are two-sided ideals for any open subset $O$ of the manifold $M$. Thus we want to transfer this 'locality structure' to the GNS representation space and use it to study the GNS representations more closely.

The main results of this work are organized as follows: After a brief summary of deformation quantization in Section 2, the first crucial observation in Section 3 is that one can assign to any vector in the GNS pre-Hilbert space a support on $M$. Hence one can think of these abstract equivalence classes as located on the underlying manifold. In particular the GNS pre-Hilbert space inherits a net structure of orthogonal subspaces indexed by the open sets of $M$ where two such subspaces are orthogonal if the corresponding open sets on the manifold are disjoint. This allows for the definition of local operators where an endomorphism of the GNS representation space is called local if it is compatible with the above net structure. It turns out that the GNS representation itself always yields such local operators. Thus we consider the commutant in the local operators of the GNS representation in order to study the question when a representation is to be regarded as a 'thermal' one or a 'pure' one (Section 3).

In Section 4 we consider faithful positive functionals and their GNS representations. It turns out that a positive functional is faithful if and only if its support is the whole manifold and a GNS representation is faithful if and only if the corresponding positive functional is faithful, a feature which is quite different from usual $C^{*}$-algebra theory.

In Section 5 we discuss particular examples of positive functionals as traces and KMS functionals on connected symplectic manifolds, $\delta$-functionals on Kähler manifolds, and Schrödinger functionals on cotangent bundles. It turns out that the abstract notion of support of vectors in the GNS preHilbert space coincides with the usual notion of support in that cases where the GNS pre-Hilbert 
space is isomorphic to spaces of formal wave functions. Furthermore in these examples the local commutant yields the physically correct characterization of 'thermal' vs. 'pure' representations.

In Section 6 we define analogues of strong and *-strong operator topologies for the local operators and arrive at an analogue of von Neumann's double commutant theorem for the cases of GNS representations with respect to a KMS functional and a Schrödinger functional, respectively. Here the completion in the strong operator topology coincides with the double commutant within the local operators.

Finally, in Section 7 we prove by a simple algebraic computation a formal analogue of the Tomita-Takesaki theorem for the local operators in a GNS representation of a KMS functional.

In Appendix $\mathrm{A}$ and $\mathrm{B}$ we briefly summarize well-known results on the formal GNS construction as well as on formal series and their $\lambda$-adic topology.

\section{Basic definitions}

In this section we recall some basic features of deformation quantization to set-up our notation and discuss the definition of the support of linear functionals.

Throughout this article $M$ denotes a symplectic or Poisson manifold endowed with a local or even differential star product $*$. Hereby a star product is a formal associative deformation of the pointwise multiplication of the smooth complex-valued functions $C^{\infty}(M)$ on $M$ in direction of the Poisson bracket. More precisely, * : $C^{\infty}(M)[[\lambda]] \times C^{\infty}(M)[[\lambda]] \rightarrow C^{\infty}(M)[[\lambda]]$ is an associative $\mathbb{C}[[\lambda]]$-bilinear product such that for $f, g \in C^{\infty}(M)$

$$
f * g=\sum_{r=0}^{\infty} \lambda^{r} M_{r}(f, g),
$$

with local or even bidifferential operators $M_{r}$ fulfilling $M_{0}(f, g)=f g$ and $M_{1}(f, g)-M_{1}(g, f)=$ i $\{f, g\}$. Moreover we require that for $r \geq 1$ the operator $M_{r}$ vanishes on constants, whence $1 * f=$ $f=f * 1$. Note that the $\mathbb{C}[[\lambda]]$-bilinearity implies the form (2.1) and in particular the $\lambda$-adically continuity of $*$, see e.g. [17, Prop. 2.1]. With this normalization the formal parameter $\lambda$ is directly to be identified with Planck's constant $\hbar$ and may be substituted in convergent situations. Since we are interested in GNS representations we need a ${ }^{*}$-involution, i.e. a $\mathbb{C}[[\lambda]]$-anti-linear involutive anti-automorphism of the star product $*$. Thus we additionally require the property

$$
\overline{f * g}=\bar{g} * \bar{f}
$$

where $f \mapsto \bar{f}$ denotes the pointwise complex conjugation and $\lambda$ is considered to be real, i.e. we define $\bar{\lambda}:=\lambda$. Note that such star products always exist.

Next we consider certain sub-algebras of $C^{\infty}(M)[[\lambda]]$ indexed by the open subsets of $M$. Viewing elements of $C^{\infty}(M)[[\lambda]]$ as $\mathbb{C}[[\lambda]]$-valued functions we define the support supp $f$ of $f=\sum_{r=0}^{\infty} \lambda^{r} f_{r} \in$ $C^{\infty}(M)[[\lambda]]$ in the usual way to be the closure of the set $\{x \in M \mid f(x) \neq 0\}$ which coincides with the closure of the set $\bigcup_{r=0}^{\infty} \operatorname{supp} f_{r}$. Note that if $\operatorname{supp} f$ is compact then supp $f_{r}$ is compact for all $r$ but the converse is not true in general. Due to the required locality of the star product we have

$$
\operatorname{supp}(f * g) \subseteq \operatorname{supp} f \cap \operatorname{supp} g
$$

for all $f, g \in C^{\infty}(M)[[\lambda]]$. Furthermore, for any open set $O \subseteq M$ the spaces $\mathcal{A}(O)=\{f \in$ $\left.C^{\infty}(M)[[\lambda]] \mid \operatorname{supp} f \subseteq O\right\}$ and $C_{0}^{\infty}(O)[[\lambda]]$ are two-sided ideals of $C^{\infty}(M)[[\lambda]]$ which are stable under complex conjugation. Clearly, if $O \subseteq O^{\prime}$ then $\mathcal{A}(O) \subseteq \mathcal{A}\left(O^{\prime}\right)$ and $C_{0}^{\infty}(O)[[\lambda]] \subseteq C_{0}^{\infty}\left(O^{\prime}\right)[[\lambda]]$, respectively. Note that $\mathcal{A}(O)$ and $C_{0}^{\infty}(O)[[\lambda]]$ in general have no unit element. Note furthermore 
that if $f \in C_{0}^{\infty}(O)[[\lambda]]$ it may happen that supp $f$ is not contained in $O$ but only in the closure of $O$. Moreover, supp $f$ needs not to be compact. This may cause some subtleties later on and hence we shall define

$$
\mathcal{A}_{0}(O):=\left\{f \in C_{0}^{\infty}(O)[[\lambda]] \mid \operatorname{supp} f \subseteq O\right\} \subseteq C_{0}^{\infty}(M)[[\lambda]]
$$

which is again a two-sided ideal in the whole algebra. All these net structures motivate to speak of a net of local observables similar to algebraic quantum field theory, see e.g. [20]. But note that here locality means locality in phase space and not in Minkowski space.

In a next step we consider $\mathbb{C}[[\lambda]]$-linear functionals $\omega: C_{0}^{\infty}(M)[[\lambda]] \rightarrow \mathbb{C}[[\lambda]]$ or $\omega: C^{\infty}(M)[[\lambda]] \rightarrow$ $\mathbb{C}[[\lambda]]$, respectively. Since in the most relevant examples we have in mind the functionals incorporate integrations over $M$ it is reasonable to consider mainly functionals defined on $C_{0}^{\infty}(M)[[\lambda]]$ only. We define the support supp $\omega$ of such a functional as usual to be the complement of the union of those open sets $O \subseteq M$ with $\left.\omega\right|_{C_{0}^{\infty}(O)[[\lambda]]}=0$. Since any $\mathbb{C}[[\lambda]]$-linear functional is automatically continuous in the $\lambda$-adic topology we obviously have $\left.\omega\right|_{C_{0}^{\infty}(O)[[\lambda]]}=0$ if and only if $\left.\omega\right|_{C_{0}^{\infty}(O)}=0$. Hence it suffices to 'test' $\omega$ on $C_{0}^{\infty}(O)$ in order to determine the support of $\omega$ and thus we have

$$
\operatorname{supp} \omega:=M \backslash \bigcup_{\left.\omega\right|_{C_{0}^{\infty}(O)[[\lambda]]}=0} O=M \backslash \bigcup_{\left.\omega\right|_{C_{0}^{\infty}(O)}=0} O
$$

where $O$ ranges over the open subsets of $M$. Then the following lemma is obtained completely analogously to the non-formal case of distributions, see e.g. [31, p. 164], and does not yet use the star product.

Lemma 2.1 Let $M$ be a manifold and let $\omega, \omega^{\prime}: C_{0}^{\infty}(M)[[\lambda]] \rightarrow \mathbb{C}[[\lambda]]$ be $\mathbb{C}[[\lambda]]$-linear functionals. Then $\operatorname{supp} \omega$ is closed and $\left.\omega\right|_{C_{0}^{\infty}(M \backslash \operatorname{supp} \omega)[[\lambda]]}=0$. If $f \in C_{0}^{\infty}(M)[[\lambda]]$ fulfills supp $f \cap \operatorname{supp} \omega=\emptyset$ then $\omega(f)=0$. Finally $\operatorname{supp}\left(\omega+\omega^{\prime}\right) \subseteq \operatorname{supp} \omega \cup \operatorname{supp} \omega^{\prime}$ and $\operatorname{supp} \alpha \omega \subseteq \operatorname{supp} \omega$ for $\alpha \in \mathbb{C}[[\lambda]]$.

Similar to the case of distributions with compact support we can construct an extension of a $\mathbb{C}[[\lambda]]$ linear functional $\omega: C_{0}^{\infty}(M)[[\lambda]] \rightarrow \mathbb{C}[[\lambda]]$ to a $\mathbb{C}[[\lambda]]$-linear functional $\hat{\omega}: C^{\infty}(M)[[\lambda]] \rightarrow \mathbb{C}[[\lambda]]$ provided the support of $\omega$ is compact.

Proposition 2.2 Let $M$ be a manifold and let $\omega: C_{0}^{\infty}(M)[[\lambda]] \rightarrow \mathbb{C}[[\lambda]]$ be a $\mathbb{C}[[\lambda]]$-linear functional with compact support. Then there exists a unique extension $\hat{\omega}: C^{\infty}(M)[[\lambda]] \rightarrow \mathbb{C}[[\lambda]]$ of $\omega$ with the property that for $f \in C^{\infty}(M)[[\lambda]]$ with $\operatorname{supp} f \cap \operatorname{supp} \omega=\emptyset$ one has $\hat{\omega}(f)=0$.

Proof: Since $\operatorname{supp} \omega$ is assumed to be compact we find a smooth partition of unity $\chi_{0}+\chi_{1}=1$ such that $\operatorname{supp} \chi_{0} \subseteq M \backslash \operatorname{supp} \omega, \chi_{1}$ has compact support, and $\chi_{1}$ is equal to 1 in an open neighborhood of $\operatorname{supp} \omega$. Then one verifies easily that $\hat{\omega}(f):=\omega\left(\chi_{1} f\right)$ is a well-defined extension having the desired properties which proves the existence. Now let $\hat{\omega}^{\prime}$ be another such extension then for all $f \in C^{\infty}(M)[[\lambda]]$ one has $\operatorname{supp}\left(\chi_{0} f\right) \cap \operatorname{supp} \omega=\emptyset$. Hence $\hat{\omega}^{\prime}(f)=\hat{\omega}^{\prime}\left(\chi_{0} f+\chi_{1} f\right)=\hat{\omega}^{\prime}\left(\chi_{1} f\right)=\omega(f)=\hat{\omega}(f)$ since $\chi_{1} f$ has compact support and clearly $\omega\left(\chi_{1} f\right)=\omega(f)$.

\section{$3 \quad$ Locality in GNS representations and commutants}

Let us now consider positive $\mathbb{C}[[\lambda]]$-linear functionals and their induced GNS representations and investigate the relations between the support of these functionals and their GNS representations. For a detailed exposition concerning the GNS construction in deformation quantization see [8, 12], and see App. A for a short summary and notation. 
Let $\omega: C_{0}^{\infty}(M)[[\lambda]] \rightarrow \mathbb{C}[[\lambda]]$ be a positive $\mathbb{C}[[\lambda]]$-linear functional. Then $\mathcal{J}_{\omega}$ denotes the Gel'fand ideal of $\omega$ and $\mathfrak{H}_{\omega}$ the GNS pre-Hilbert space. Note that the GNS representation $\pi_{\omega}$ : $C_{0}^{\infty}(M)[[\lambda]] \rightarrow \mathfrak{B}\left(\mathfrak{H}_{\omega}\right)$ extends to the whole algebra $C^{\infty}(M)[[\lambda]]$ since $C_{0}^{\infty}(M)[[\lambda]]$ is a two-sided ideal in $C^{\infty}(M)[[\lambda]]$ stable under complex conjugation, see [12, Cor. 1].

The crucial observation is that we can associate to any vector $\psi_{f}$ in the GNS pre-Hilbert space $\mathfrak{H}_{\omega}$ a 'support' $\operatorname{supp} \psi_{f}$ by

$$
\operatorname{supp} \psi_{f}:=\operatorname{supp} \omega_{f}, \quad \text { where } \quad \omega_{f}(g):=\left\langle\psi_{f}, \pi_{\omega}(g) \psi_{f}\right\rangle .
$$

Here $f, g \in C_{0}^{\infty}(M)[[\lambda]]$. Note that the $\mathbb{C}[[\lambda]]$-linear functional $\omega_{f}$ is positive and explicitly given by

$$
\omega_{f}(g)=\omega(\bar{f} * g * f) .
$$

Clearly $\omega_{f}$ depends only on the equivalence class $\psi_{f}$. A first characterization of this support is given by the following lemma:

Lemma 3.1 Let $\omega: C_{0}^{\infty}(M)[[\lambda]] \rightarrow \mathbb{C}[[\lambda]]$ be a positive $\mathbb{C}[[\lambda]]$-linear functional, $f, g \in C_{0}^{\infty}(M)[[\lambda]]$, and $\alpha \in \mathbb{C}[[\lambda]]$.

i.) If $\operatorname{supp} f \cap \operatorname{supp} \omega=\emptyset$ then $f \in \mathcal{J}_{\omega}$ and thus $\psi_{f}=0$.

ii.) $\operatorname{supp} \psi_{f} \subseteq \operatorname{supp} f \cap \operatorname{supp} \omega$.

iii.) $\operatorname{supp}\left(\psi_{f}+\psi_{g}\right) \subseteq \operatorname{supp} \psi_{f} \cup \operatorname{supp} \psi_{g}$ and $\operatorname{supp} \alpha \psi_{f} \subseteq \operatorname{supp} \psi_{f}$.

Proof: Let $\operatorname{supp} f \cap \operatorname{supp} \omega=\emptyset$ then also $\operatorname{supp}(\bar{f} * f) \cap \operatorname{supp} \omega=\emptyset$ whence $\omega(\bar{f} * f)=0$ due to Lemma 2.1 which proves the first part. To avoid trivialities assume $\operatorname{supp} f \cap \operatorname{supp} \omega \neq \emptyset$. Considering $g \in C_{0}^{\infty}(M \backslash(\operatorname{supp} f \cap \operatorname{supp} \omega))$ we find $\operatorname{supp}(\bar{f} * g * f) \cap \operatorname{supp} \omega=\emptyset$ whence $\omega_{f}(g)=0$ again due to Lemma 2.1. Thus the second part follows. Finally we observe that for $h \in C_{0}^{\infty}\left(M \backslash\left(\operatorname{supp} \psi_{f} \cup \operatorname{supp} \psi_{g}\right)\right)$ we have $\omega(\bar{f} * h * g) \overline{\omega(\bar{f} * h * g)} \leq \omega(\bar{f} * f) \omega(\bar{g} * \bar{h} * h * g)=0$ by the Cauchy-Schwarz inequality since $\operatorname{supp} h \cap \operatorname{supp} \psi_{g}=\emptyset$. Hence we have $\omega(\bar{f} * h * g)=0$ and similar we find $\omega(\bar{g} * h * f)=0$. Now the third part follows easily since for such $h$ one finds $\omega_{f+g}(h)=\omega_{f}(h)+\omega_{g}(h)+\omega(\bar{f} * h * g)+\omega(\bar{g} * h * f)=0$.

Note that in general $\operatorname{supp} \psi_{f}$ is strictly smaller than $\operatorname{supp} f \cap \operatorname{supp} \omega$. Examples can easily be found e.g. in the Schrödinger representations in Section 5.

As a first consequence of this lemma we observe that for any open set $O \subseteq M$ the space

$$
\mathfrak{H}_{\omega}(O):=\left\{\psi_{f} \in \mathfrak{H}_{\omega} \mid \operatorname{supp} \psi_{f} \subseteq O\right\}
$$

is a sub-module of $\mathfrak{H}_{\omega}$ and clearly $\mathfrak{H}_{\omega}(O) \subseteq \mathfrak{H}_{\omega}\left(O^{\prime}\right)$ for $O \subseteq O^{\prime}$. Thus the net structure of the observable algebra $C_{0}^{\infty}(M)[[\lambda]]$ induces a net structure for the GNS pre-Hilbert space $\mathfrak{H}_{\omega}$. Note that it may happen that $\mathfrak{H}_{\omega}(O)=\{0\}$ for certain open $O \subseteq M$. In order to characterize this net of pre-Hilbert spaces $\mathfrak{H}_{\omega}(O)$ we need the following lemma.

Lemma 3.2 Let $\omega: C_{0}^{\infty}(M)[[\lambda]] \rightarrow \mathbb{C}[[\lambda]]$ be a positive $\mathbb{C}[[\lambda]]$-linear functional and let $\psi_{f}, \psi_{g} \in \mathfrak{H}_{\omega}$.

i.) If $\operatorname{supp} \psi_{f} \subseteq O$ with $O \subseteq M$ open then there exists a $\tilde{f} \in \mathcal{A}_{0}(O) \subseteq C_{0}^{\infty}(O)[[\lambda]]$ such that $\psi_{f}=\psi_{\tilde{f}}$, i.e. $f-\tilde{f} \in \mathcal{J}_{\omega}$.

ii.) $\operatorname{supp} \psi_{f} \cap \operatorname{supp} \psi_{g}=\emptyset$ implies $\left\langle\psi_{f}, \psi_{g}\right\rangle=0$. 
Proof: Choose an open neighborhood $U$ of $M \backslash O$ such that $U \cap \operatorname{supp} \psi_{f}=\emptyset$. Then let $\chi_{0}+\chi_{1}=1$ be a smooth partition of unity subordinate to the cover $U \cup O=M$, i.e. $\operatorname{supp} \chi_{0} \subseteq U$ and $\operatorname{supp} \chi_{1} \subseteq O$. Since $\left\langle\psi_{\chi_{0} * f}, \psi_{\chi_{0} * f}\right\rangle=\omega_{f}\left(\bar{\chi}_{0} * \chi_{0}\right)=0$ due to $\operatorname{supp} \chi_{0} \cap \operatorname{supp} \psi_{f}=\emptyset$ and Lemma 2.1 one obtains $\psi_{\chi_{0} * f}=0$ whence $\psi_{f}=\psi_{\chi_{1} * f}$. Setting $\tilde{f}=\chi_{1} * f$ the first part follows since clearly supp $\tilde{f} \subseteq \operatorname{supp} \chi_{1} \subseteq O$. Secondly, choose two open sets $O, O^{\prime}$ such that $O \cap O^{\prime}=\emptyset$ and $\operatorname{supp} \psi_{f} \subseteq O$ as well as supp $\psi_{g} \subseteq O^{\prime}$. Due to the first part we may thus assume without restrictions that $f \in \mathcal{A}_{0}(O)$ and $g \in \mathcal{A}_{0}\left(O^{\prime}\right)$. Then $\bar{f} * g=0$ whence $\left\langle\psi_{f}, \psi_{g}\right\rangle=\omega(\bar{f} * g)=0$.

This lemma suggests to consider those functions in the Gel'fand ideal $\mathcal{J}_{\omega}$ having their support in some open set $O$. We define

$$
\mathcal{J}_{\omega}(O):=\mathcal{A}_{0}(O) \cap \mathcal{J}_{\omega}
$$

for open $O \subseteq M$. Here we use $\mathcal{A}_{0}(O)$ instead of $C_{0}^{\infty}(O)[[\lambda]]$ and this difference will be crucial for the next proposition. By definition $\mathcal{J}_{\omega}(O) \subseteq \mathcal{A}_{0}(O)$ holds. Hence we can compare $\mathfrak{H}_{\omega}(O)$ with the quotient $\mathcal{A}_{0}(O) / \mathcal{J}_{\omega}(O)$ which carries a natural pre-Hilbert structure induced by the restriction of $\omega$ to $\mathcal{A}_{0}(O)$. The next proposition states that they are canonically isomorphic.

Proposition 3.3 Let $\omega: C_{0}^{\infty}(M)[[\lambda]] \rightarrow \mathbb{C}[[\lambda]]$ be a positive $\mathbb{C}[[\lambda]]$-linear functional and $O, O^{\prime} \subseteq M$ open.

i.) $\mathfrak{H}_{\omega}(O)$ is canonically isometric to $\mathcal{A}_{0}(O) / \mathcal{J}_{\omega}(O)$.

ii.) If $O \cap O^{\prime}=\emptyset$ then $\mathfrak{H}_{\omega}(O) \perp \mathfrak{H}_{\omega}\left(O^{\prime}\right)$.

iii.) If $O \cap \operatorname{supp} \omega=\emptyset$ then $\mathfrak{H}_{\omega}(O)=\{0\}$.

Proof: Denote by $[f] \in \mathcal{A}_{0}(O) / \mathcal{J}_{\omega}(O)$ the equivalence class of $f \in \mathcal{A}_{0}(O)$. Then the Hermitian product of $[f]$ and $[g]$ is given by $\langle[f],[g]\rangle=\omega(\bar{f} * g)$ and the canonical isomorphism to $\mathfrak{H}_{\omega}(O)$ is given by $[f] \mapsto \psi_{f}$. Since $\operatorname{supp} \psi_{f} \subseteq \operatorname{supp} f \subseteq O$ this is clearly well-defined and isometric, hence injective. The surjectivity follows from Lemma 3.2 (i). The second part follows directly from Lemma 3.2 (ii). Finally let $O \cap \operatorname{supp} \omega=\emptyset$ and $\psi_{f} \in \mathfrak{H}_{\omega}(O)$. Then we can assume $f \in C_{0}^{\infty}(O)[[\lambda]]$ due to Lemma 3.2 (i) whence $\left\langle\psi_{f}, \psi_{f}\right\rangle=\omega(\bar{f} * f)=0$ due to Lemma 2.1. Hence $\psi_{f}=0$, proving the third part.

As a first application of this proposition we have the following corollary concerning convex sums of positive functionals:

Corollary 3.4 Let $\omega_{1}, \omega_{2}: C_{0}^{\infty}(M)[[\lambda]] \rightarrow \mathbb{C}[[\lambda]]$ be two positive $\mathbb{C}[[\lambda]]$-linear functionals and let $\alpha_{1}, \alpha_{2} \in \mathbb{R}[[\lambda]]$ be positive and let $\omega:=\alpha_{1} \omega_{1}+\alpha_{2} \omega_{2}$.

i.) $\operatorname{supp} \omega=\operatorname{supp} \omega_{1} \cup \operatorname{supp} \omega_{2}$.

ii.) If for $i=1,2$ one has $\operatorname{supp} \omega_{i} \subset O_{i}$ with $O_{1} \cap O_{2}=\emptyset$ and $O_{1}, O_{2}$ open, then

$$
\mathfrak{H}_{\omega}=\mathfrak{H}_{\omega}\left(O_{1} \cup O_{2}\right)=\mathfrak{H}_{\omega}\left(O_{1}\right) \oplus \mathfrak{H}_{\omega}\left(O_{2}\right)
$$

and canonically $\mathfrak{H}_{\omega}\left(O_{i}\right) \cong \mathfrak{H}_{\omega_{i}}$.

Proof: For the first part the inclusion ' $\subseteq$ ' follows from Lemma 2.1 hence consider ' $\supseteq$ '. Assume $\omega_{i}(h) \neq 0$ for some $i$ and some $h \in C_{0}^{\infty}(O)$. Choosing some $\chi \in C_{0}^{\infty}(M)$ with $\chi=1$ in an open neighborhood of supp $h$ one has $h=\chi * h$ and thus by the Cauchy-Schwarz inequality one finds $\omega_{i}(\bar{h} * h)>0$. Then $\omega(\bar{h} * h) \geq \alpha_{i} \omega_{i}(\bar{h} * h)>0$ shows $\omega(\bar{h} * h) \neq 0$. Thus the first part follows since $\operatorname{supp}(\bar{h} * h) \subseteq \operatorname{supp} h$. 
Secondly, consider $O_{1} \cup O_{2}$ then $\mathfrak{H}_{\omega}=\mathfrak{H}_{\omega}\left(O_{1} \cup O_{2}\right)$ since $\operatorname{supp} \psi_{f} \subseteq \operatorname{supp} \omega \subseteq O_{1} \cup O_{2}$ for all $\psi_{f} \in \mathfrak{H}_{\omega}$ by Lemma 3.1 (ii). Furthermore we may assume for $\psi_{f}$ that $f \in \mathcal{A}_{0}\left(O_{1} \cup O_{2}\right)$ due to Lemma 3.2 (i). Clearly any such $f$ can be uniquely written as $f=f_{1}+f_{2}$ where supp $f_{i} \subseteq O_{i}$ since $O_{1} \cap O_{2}=\emptyset$. But then $\psi_{f}=\psi_{f_{1}}+\psi_{f_{2}}$ with supp $\psi_{f_{i}} \subseteq O_{i}$. This induces the above direct sum. Finally, the canonical isomorphisms $\mathfrak{H}_{\omega}\left(O_{i}\right) \cong \mathfrak{H}_{\omega_{i}}$ are induced by the identity map which follows again from Lemma 3.2.

Since the GNS pre-Hilbert space $\mathfrak{H}_{\omega}$ inherits the net structure $\mathfrak{H}_{\omega}(O)$ from the topology of $M$ for any positive $\mathbb{C}[[\lambda]]$-linear functional $\omega$ we are particularly interested in endomorphisms of $\mathfrak{H}_{\omega}$ respecting this locality structure. This motivates the following definition of local operators.

Definition 3.5 Let $\omega: C_{0}^{\infty}(M)[[\lambda]] \rightarrow \mathbb{C}[[\lambda]]$ be a positive $\mathbb{C}[[\lambda]]$-linear functional and $A: \mathfrak{H}_{\omega} \rightarrow \mathfrak{H}_{\omega}$ $a \mathbb{C}[[\lambda]]$-linear map. Then $A$ is called local if for all $\psi_{f} \in \mathfrak{H}_{\omega}$

$$
\operatorname{supp} A \psi_{f} \subseteq \operatorname{supp} \psi_{f}
$$

The set of local $\mathbb{C}[[\lambda]]$-linear endomorphisms of $\mathfrak{H}_{\omega}$ is denoted by $\mathfrak{L}\left(\mathfrak{H}_{\omega}\right)$.

Similarly one defines the local $\mathbb{C}[[\lambda]]$-linear maps $\mathfrak{L}\left(\mathfrak{H}_{\omega}, \mathfrak{H}_{\omega^{\prime}}\right)$ from one GNS pre-Hilbert space $\mathfrak{H}_{\omega}$ into another $\mathfrak{H}_{\omega^{\prime}}$, where $\omega$ and $\omega^{\prime}$ are two positive $\mathbb{C}[[\lambda]]$-linear functionals. The following proposition is an obvious consequence of the preceding lemmas.

Proposition 3.6 Let $\omega: C_{0}^{\infty}(M)[[\lambda]] \rightarrow \mathbb{C}[[\lambda]]$ be a positive $\mathbb{C}[[\lambda]]$-linear functional then $\mathfrak{L}\left(\mathfrak{H}_{\omega}\right)$ is a subalgebra of all endomorphisms of $\mathfrak{H}_{\omega}$.

The abstract notion of local operators in GNS pre-Hilbert spaces turns out to be fundamental for the whole following discussion. In fact the GNS representation always is local.

Theorem 3.7 Let $(M, *)$ be a Poisson manifold with local star product and let $\omega: C_{0}^{\infty}(M)[[\lambda]] \rightarrow$ $\mathbb{C}[[\lambda]]$ be a positive $\mathbb{C}[[\lambda]]$-linear functional with $G N S$ pre-Hilbert space $\mathfrak{H}_{\omega}$. Then for any $f \in$ $C^{\infty}(M)[[\lambda]]$ the operator $\pi_{\omega}(f): \mathfrak{H}_{\omega} \rightarrow \mathfrak{H}_{\omega}$ is local. Moreover,

$$
\operatorname{supp} \pi_{\omega}(f) \psi_{g} \subseteq \operatorname{supp} f \cap \operatorname{supp} \psi_{g}
$$

for all $f \in C^{\infty}(M)[[\lambda]]$ and $\psi_{g} \in \mathfrak{H}_{\omega}$.

Proof: We have to determine the support of $\psi_{f * g}$. Without restriction assume supp $\psi_{g} \neq M$ and consider $h \in C_{0}^{\infty}\left(M \backslash \operatorname{supp} \psi_{g}\right)$. Then $\left\langle\psi_{f * g}, \pi_{\omega}(h) \psi_{f * g}\right\rangle=\omega(\bar{g} * \bar{f} * h * f * g)=\omega_{g}(\bar{f} * h * f)=0$ since $\operatorname{supp}(\bar{f} * h * f) \subseteq \operatorname{supp} h$. Thus supp $\psi_{f * g} \subseteq \psi_{g}$ follows. Moreover, supp $\pi_{\omega}(f) \psi_{g} \subseteq \operatorname{supp} \omega \cap \operatorname{supp} g \cap \operatorname{supp} f$ due to Lemma 3.1 (ii) and the locality of $*$. Thus (3.7) follows.

We shall now turn to the question how these local operators can be used to give a reasonable distinction between the various types of GNS representations. In particular we are searching for a suitable way to distinguish 'thermal' representations from 'pure' ones. In the usual $C^{*}$-algebra theory as used e.g. in quantum mechanics and quantum field theory the concept of 'thermal' and 'pure' states is highly developed both from the physical and mathematical point of view and can be summarized as follows. Pure states are the extremal points in the convex set of all states and a state is pure if and only if its GNS representation is irreducible which is the case if and only if the commutant in the GNS representation is trivial, see e.g. [14, Thm. 2.3.19]. On the other hand the so-called KMS states, which are understood to describe 'thermal' behavior, are not pure states. In deformation quantization it will turn out that the commutant of the GNS representation within the local operators is the appropriate choice for the definition of a commutant which distinguishes 'thermal' from 'pure' representations.

Before we investigate local commutants let us firstly consider the adjoints of local operators. 
Lemma 3.8 Let $\omega: C_{0}^{\infty}(M)[[\lambda]] \rightarrow \mathbb{C}[[\lambda]]$ be a positive $\mathbb{C}[[\lambda]]$-linear functional with GNS preHilbert space $\mathfrak{H}_{\omega}$. If $A \in \mathfrak{L}\left(\mathfrak{H}_{\omega}\right)$ has an adjoint operator $A^{*}$ then $A^{*}$ is local again.

Proof: Assume $A^{*}$ exists and let $\psi_{f} \in \mathfrak{H}_{\omega}$ be arbitrary where we may assume supp $\psi_{f} \neq M$ to avoid trivialities. Choose $h \in C_{0}^{\infty}(M)[[\lambda]]$ such that $A^{*} \psi_{f}=\psi_{h}$. Then it is sufficient to show $\left.\omega_{h}\right|_{C_{0}^{\infty}\left(M \backslash \operatorname{supp} \psi_{f}\right)}=0$ since in this case $\operatorname{supp} A^{*} \psi_{f}=\operatorname{supp} \psi_{h} \subseteq \operatorname{supp} \psi_{f}$. Hence let $g \in C_{0}^{\infty}\left(M \backslash \operatorname{supp} \psi_{f}\right)$ then we compute $\omega_{h}(g)$ using $\left(A^{*}\right)^{*}=A$ and $\pi_{\omega}(g)^{*}=\pi_{\omega}(\bar{g})$ and obtain $\omega_{h}(g)=\left\langle A \pi_{\omega}(\bar{g}) A^{*} \psi_{f}, \psi_{f}\right\rangle$. Now $\pi_{\omega}(\bar{g})$ is local and due to (3.7) we even have $\operatorname{supp} \pi_{\omega}(\bar{g}) A^{*} \psi_{f} \subseteq \operatorname{supp} g$. With $\operatorname{supp} g \cap \operatorname{supp} \psi_{f}=\emptyset$ we conclude that $\omega_{h}(g)=0$ using Lemma 2.1. Thus $A^{*}$ is local.

It will turn out that in various examples the local operators automatically have an adjoint though this is not evident from the abstract point of view. We now define

$$
\mathfrak{L} \mathfrak{B}\left(\mathfrak{H}_{\omega}\right):=\mathfrak{L}\left(\mathfrak{H}_{\omega}\right) \cap \mathfrak{B}\left(\mathfrak{H}_{\omega}\right)
$$

and conclude from the last lemma that $\mathfrak{L} \mathfrak{B}\left(\mathfrak{H}_{\omega}\right)$ is a *-algebra.

Proposition 3.9 Let $\omega: C_{0}^{\infty}(M)[[\lambda]] \rightarrow \mathbb{C}[[\lambda]]$ be a positive $\mathbb{C}[[\lambda]]$-linear functional with GNS pre-Hilbert space $\mathfrak{H}_{\omega}$. Then $\mathfrak{L} \mathfrak{B}\left(\mathfrak{H}_{\omega}\right)$ is a ${ }^{*}$-algebra over $\mathbb{C}[[\lambda]]$.

Using the algebras $\mathfrak{L}\left(\mathfrak{H}_{\omega}\right)$ and $\mathfrak{L} \mathfrak{B}\left(\mathfrak{H}_{\omega}\right)$, respectively, we can define the commutant of an arbitrary subset of $\mathfrak{L}\left(\mathfrak{H}_{\omega}\right)$ and $\mathfrak{L} \mathfrak{B}\left(\mathfrak{H}_{\omega}\right)$, respectively, in the following way:

Definition 3.10 Let $\omega: C_{0}^{\infty}(M)[[\lambda]] \rightarrow \mathbb{C}[[\lambda]]$ be a positive $\mathbb{C}[[\lambda]]$-linear functional with $G N S$ pre-Hilbert space $\mathfrak{H}_{\omega}$ and let $\mathcal{B} \subseteq \mathfrak{L}\left(\mathfrak{H}_{\omega}\right)$ or $\mathcal{B} \subseteq \mathfrak{L} \mathfrak{B}\left(\mathfrak{H}_{\omega}\right)$ then

$$
\begin{aligned}
\mathcal{B}_{\mathfrak{L}}^{\prime} & :=\left\{A \in \mathfrak{L}\left(\mathfrak{H}_{\omega}\right) \mid \forall B \in \mathcal{B}: A B=B A\right\}, \\
\mathcal{B}_{\mathfrak{L} \mathfrak{B}}^{\prime} & :=\left\{A \in \mathfrak{L} \mathfrak{B}\left(\mathfrak{H}_{\omega}\right) \mid \forall B \in \mathcal{B}: A B=B A\right\}
\end{aligned}
$$

are called the local and ${ }^{*}$-local commutant of $\mathcal{B}$ in $\mathfrak{L}\left(\mathfrak{H}_{\omega}\right)$ and $\mathfrak{L} \mathfrak{B}\left(\mathfrak{H}_{\omega}\right)$, respectively.

In principle one could also define a $\mathfrak{B}$-commutant in $\mathfrak{B}\left(\mathfrak{H}_{\omega}\right)$ but this will not be as useful as the above versions of commutants. If the context is clear we will sometimes omit the subscript $\mathfrak{L}$ resp. $\mathfrak{L} \mathfrak{B}$. Now let $\mathcal{B} \subseteq \mathfrak{L}\left(\mathfrak{H}_{\omega}\right)$ or $\mathfrak{L} \mathfrak{B}\left(\mathfrak{H}_{\omega}\right)$, respectively. Then $\mathcal{B}^{\prime}$ is a subalgebra of $\mathfrak{L}\left(\mathfrak{H}_{\omega}\right)$ or $\mathfrak{L} \mathfrak{B}\left(\mathfrak{H}_{\omega}\right)$, respectively, and if $\mathcal{B}=\mathcal{B}^{*} \subseteq \mathfrak{L} \mathfrak{B}\left(\mathfrak{H}_{\omega}\right)$ then $\mathcal{B}_{\mathfrak{L} \mathfrak{B}}^{\prime}$ is even a ${ }^{*}$-subalgebra. Note that $\mathcal{B}$ needs not to be an algebra at all. Furthermore one has $\mathcal{B} \subseteq \mathcal{B}^{\prime \prime}$ and if $\mathcal{C} \subseteq \mathcal{B}$ then $\mathcal{B}^{\prime} \subseteq \mathcal{C}^{\prime}$. Hence $\mathcal{B}^{\prime \prime \prime}=\mathcal{B}^{\prime}$ and thus the commutant of a set is always closed under taking the double commutant. Note finally that for a subalgebra $\mathcal{B}$ the algebra $\mathcal{Z}=\mathcal{B} \cap \mathcal{B}^{\prime}$ is the center of $\mathcal{B}$ and $\mathcal{B}^{\prime}$. As for complex Hilbert spaces we shall call a ${ }^{*}$-subalgebra of $\mathfrak{L} \mathfrak{B}\left(\mathfrak{H}_{\omega}\right)$ a von Neumann algebra if $\mathcal{B}=\mathcal{B}_{\mathfrak{L} \mathfrak{B}}^{\prime \prime}$ and a factor if in addition the center is trivial. Here we are forced to take $\mathfrak{L} \mathfrak{B}$-commutants since we are interested in ${ }^{*}$-algebras.

Now we can use this notion of local commutants to characterize 'pure' and 'thermal' GNS representations. A first result is obtained in the following proposition which can be viewed as an analogue to the well-known situation of mixed states for complex $C^{*}$-algebras, see e.g. [14, Thm. 2.3.19].

Proposition 3.11 Let $\omega_{1}, \omega_{2}: C_{0}^{\infty}(M)[[\lambda]] \rightarrow \mathbb{C}[[\lambda]]$ be two non-zero positive $\mathbb{C}[[\lambda]]$-linear functionals with $\operatorname{supp} \omega_{1} \cap \operatorname{supp} \omega_{2}=\emptyset$. Then the local (and ${ }^{*}$-local) commutant of $\pi_{\omega}(\mathcal{A})$ is non-trivial, where $\omega=\alpha_{1} \omega_{1}+\alpha_{2} \omega_{2}$, provided $\alpha_{1}, \alpha_{2}>0$. 
Proof: Since supp $\omega_{1} \cap \operatorname{supp} \omega_{2}=\emptyset$ we can find two open sets $O_{1}, O_{2}$ such that supp $\omega_{i} \subseteq O_{i}$ and $O_{1} \cap O_{2}=\emptyset$. Now we apply Cor. 3.4 to obtain $\mathfrak{H}_{\omega}=\mathfrak{H}_{\omega}\left(O_{1}\right) \oplus \mathfrak{H}_{\omega}\left(O_{2}\right)$. Then clearly the projectors on $\mathfrak{H}_{\omega}\left(O_{i}\right), i=1,2$, commute with $\pi_{\omega}(f)$ for all $f \in \mathcal{A}$ and are clearly ${ }^{*}$-local operators proving the proposition.

Note that with the hypothesis of the above proposition any function $\chi \in C^{\infty}(M)[[\lambda]]$ with $\left.\chi\right|_{O_{i}}=c_{i}$, where $c_{i} \in \mathbb{C}[[\lambda]]$ are constants, is in the center of $\pi_{\omega}\left(C^{\infty}(M)[[\lambda]]\right)$ but acts non-trivial on $\mathfrak{H}_{\omega}$ if $c_{1} \neq c_{2}$.

Corollary 3.12 Let $\omega: C_{0}^{\infty}(M)[[\lambda]] \rightarrow \mathbb{C}[[\lambda]]$ be a positive $\mathbb{C}[[\lambda]]$-linear functional such that $\operatorname{supp} \omega$ has at least two connected components. Then $\pi_{\omega}\left(C^{\infty}(M)[[\lambda]]\right)$ has a non-trivial local (and ${ }^{*}$-local) commutant.

\section{Faithful positive linear functionals}

Let us now consider faithful positive $\mathbb{C}[[\lambda]]$-linear functionals and their GNS representations. It turns out that they can completely be characterized by their support.

First recall that a positive $\mathbb{C}[[\lambda]]$-linear functional $\omega$ is called faithful if $\mathcal{J}_{\omega}=\{0\}$. Hence the GNS pre-Hilbert space $\mathfrak{H}_{\omega}$ is canonically isomorphic to $C_{0}^{\infty}(M)[[\lambda]]$ via $\mathfrak{H}_{\omega} \ni \psi_{f} \mapsto f \in$ $C_{0}^{\infty}(M)[[\lambda]]$ as $\mathbb{C}[[\lambda]]$-modules. It will sometimes be useful not to identify $\mathfrak{H}_{\omega}$ and $C_{0}^{\infty}(M)[[\lambda]]$ but use this isomorphism since $\mathfrak{H}_{\omega}$ has the Hermitian product as additional structure. Under the above isomorphism the corresponding GNS representation is simply given by left multiplication in $C_{0}^{\infty}(M)[[\lambda]]$. Thus we use also the notion $\mathrm{L}_{f}$ instead of $\pi_{\omega}(f)$ for this particular representation where $f \in C^{\infty}(M)[[\lambda]]$. On the other hand a representation $\pi$ is called faithful if it is injective.

The following technical lemma concerning local left inverses is proved by the usual recursion techniques.

Lemma 4.1 Let $(M, *)$ be a Poisson manifold with local star product, $f=\sum_{r=0}^{\infty} \lambda^{r} f_{r}$ with $f_{r} \in$ $C_{0}^{\infty}(O)$, and $\emptyset \neq U \subseteq O$ an open subset such that $f_{0}(x) \neq 0$ for all $x \in U$. Then there exists another non-empty open subset $U^{\prime} \subseteq U$ and a function $f^{-1} \in C_{0}^{\infty}(U)[[\lambda]]$ such that $\left.f^{-1} * f\right|_{U^{\prime}}=1$.

Similarly there exist local right inverses such that $\left.f * \tilde{f}^{-1}\right|_{U^{\prime \prime}}=1$ and on $U^{\prime} \cap U^{\prime \prime}$ left and right inverses coincide. Using such a local left inverse the following proposition can be shown easily.

Proposition 4.2 Let $\omega: C_{0}^{\infty}(M)[[\lambda]] \rightarrow \mathbb{C}[[\lambda]]$ be a positive $\mathbb{C}[[\lambda]]$-linear functional. Then $\omega$ is faithful if and only if $\operatorname{supp} \omega=M$.

Proof: Let $\omega$ be faithful and let $0 \neq f \in C_{0}^{\infty}(O)$ for some non-empty open set $O$. Then $\operatorname{supp}(\bar{f} * f) \subseteq O$ and thus $\omega(\bar{f} * f)>0$. This implies $\operatorname{supp} \omega=M$. Now assume $\operatorname{supp} \omega=M$, and assume we have found a function $0 \neq f \in C_{0}^{\infty}(O)[[\lambda]]$ such that $\omega(\bar{f} * f)=0$. Without restriction we can assume that already the lowest order of $f$ is non-zero. Hence there is a non-empty open subset $U$ and a local left inverse $f^{-1} \in C_{0}^{\infty}(O)[[\lambda]]$ with $\left.f^{-1} * f\right|_{U}=1$. Now let $h \in C_{0}^{\infty}(U)[[\lambda]]$ be arbitrary then clearly $h=g * f$ with some $g \in C_{0}^{\infty}(U)[[\lambda]]$, namely $g=h * f^{-1}$. Then $\omega(h) \overline{\omega(h)} \leq \omega(\bar{g} * g) \omega(\bar{f} * f)=0$ shows $\omega(h)=0$ for all $h \in C_{0}^{\infty}(U)[[\lambda]]$. Thus $\left.\omega\right|_{C_{0}^{\infty}(U)[[\lambda]]}=0$ in contradiction to $\operatorname{supp} \omega=M$.

Let us now investigate the support of $\psi_{f} \in \mathfrak{H}_{\omega}$ for a faithful positive functional $\omega$. Since in this case canonically $\mathfrak{H}_{\omega} \cong C_{0}^{\infty}(M)[[\lambda]]$ as $\mathbb{C}[[\lambda]]$-modules we expect that the support of $\psi_{f}$ coincides with the support of $f$. This is indeed the case.

Lemma 4.3 Let $\omega: C_{0}^{\infty}(M)[[\lambda]] \rightarrow \mathbb{C}[[\lambda]]$ be a faithful positive $\mathbb{C}[[\lambda]]$-linear functional. Then for all $f \in C_{0}^{\infty}(M)[[\lambda]]$ we have $\operatorname{supp} \psi_{f}=\operatorname{supp} f$. 
Proof: The inclusion $\subseteq$ is in general true due to Lemma 3.1 (ii). Hence let $f \in C_{0}^{\infty}(M)[[\lambda]]$ and assume $\operatorname{supp} \psi_{f} \neq M$ to avoid trivialities. Then for all $g \in C_{0}^{\infty}\left(M \backslash \operatorname{supp} \psi_{f}\right)$ we have $0=\omega_{f}(\bar{g} * g)=\omega(\overline{(g * f)} *(g * f))$ whence $g * f=0$ since $\omega$ is faithful. But this implies supp $f \cap\left(M \backslash \operatorname{supp} \psi_{f}\right)=\emptyset$ and thus the claim follows.

Since a faithful positive functional $\omega$ has Gel'fand ideal $\mathcal{J}_{\omega}=\{0\}$ the right multiplication $\mathrm{R}_{f}$ by $f \in C^{\infty}(M)[[\lambda]]$

$$
\mathrm{R}_{f} \psi_{g}:=\psi_{g * f}, \quad \psi_{g} \in \mathfrak{H}_{\omega}
$$

is well-defined for all $f \in C^{\infty}(M)[[\lambda]]$ and clearly again a local operator. Note that in general $\mathrm{R}_{f}$ is well-defined if $f$ is contained in the Lie idealizer of the Gel'fand ideal, i.e. the largest subalgebra of $C^{\infty}(M)[[\lambda]]$ containing $\mathcal{J}_{\omega}$ as a two-sided ideal. Using this right multiplication and the preceding results we obtain immediately the following corollaries:

Corollary 4.4 Let $\omega: C_{0}^{\infty}(M)[[\lambda]] \rightarrow \mathbb{C}[[\lambda]]$ be a faithful positive $\mathbb{C}[[\lambda]]$-linear functional. Then an operator $A: \mathfrak{H}_{\omega} \rightarrow \mathfrak{H}_{\omega}$ is local if and only if the corresponding operator $A: C_{0}^{\infty}(M)[[\lambda]] \rightarrow$ $C_{0}^{\infty}(M)[[\lambda]]$ is local in the usual sense.

Corollary 4.5 Let $\omega: C_{0}^{\infty}(M)[[\lambda]] \rightarrow \mathbb{C}[[\lambda]]$ be a faithful positive $\mathbb{C}[[\lambda]]$-linear functional. Then the local commutant of $\pi_{\omega}\left(C^{\infty}(M)[[\lambda]]\right)$ contains all right multiplications $\mathrm{R}_{f}$ with $f \in C^{\infty}(M)[[\lambda]]$. Thus $\pi_{\omega}\left(C^{\infty}(M)[[\lambda]]\right)_{\mathfrak{L}}^{\prime}$ is non-trivial (if $\operatorname{dim} M>0$ ).

The question whether the *-local commutant is non-trivial seems to be more complicated since $\operatorname{supp} \omega=M$ still allows rather 'wild' functionals whence existence of an adjoint of $\mathrm{R}_{f}$ is not obvious. Take e.g. in zeroth order a faithful positive linear functional, as e.g. integration over some positive density, then one can add in higher orders of $\lambda$ any real linear functionals and still has a positive linear functional. Nevertheless things become simpler if we consider KMS functionals in Section 5 .

Let us finally consider the GNS representation of such faithful functionals:

Proposition 4.6 Let $\omega: C_{0}^{\infty}(M)[[\lambda]] \rightarrow \mathbb{C}[[\lambda]]$ be a positive $\mathbb{C}[[\lambda]]$-linear functional with $G N S$ representation $\pi_{\omega}$. Then $\pi_{\omega}$ is faithful if and only if $\omega$ is faithful.

Proof: Note that $\pi_{\omega}$ is always understood to be extended to $C^{\infty}(M)[[\lambda]]$. Assume first that $\omega$ is faithful then $C^{\infty}(M)[[\lambda]]$ is represented on $\mathfrak{H}_{\omega} \cong C_{0}^{\infty}(M)[[\lambda]]$ by left multiplications which is clearly faithful. On the other hand assume that $\omega$ is not faithful. Then $O=M \backslash \operatorname{supp} \omega$ is a non-empty open subset due to Prop. 4.2. Due to (3.7) we have $\pi_{\omega}(f)=0$ for all $f \in C_{0}^{\infty}(O)$. Thus $\pi_{\omega}$ cannot be faithful.

We observe that if a GNS representation is faithful then it is equivalent to the left multiplication of elements of $C^{\infty}(M)[[\lambda]]$ on $C_{0}^{\infty}(M)[[\lambda]]$. Hence a faithful GNS representation $\pi_{\omega}$ does not really depend on $\omega$ but is uniquely given. Nevertheless the Hermitian product of $\mathfrak{H}_{\omega}$ still depends crucially on $\omega$. Moreover, a faithful GNS representation has always a non-trivial local commutant. Thus in this point the situation is quite different from the usual $C^{*}$-algebra theory where a faithful GNS representation can of course be irreducible, as e.g. the standard representation of the bounded operators on a Hilbert space.

\section{Basic examples}

\section{Traces and KMS functionals}

As first basic example we consider positive traces and KMS functionals. A trace tr of the algebra $C^{\infty}(M)[[\lambda]]$ is a $\mathbb{C}[[\lambda]]$-linear functional defined on $C_{0}^{\infty}(M)[[\lambda]]$ such that $\operatorname{tr}(f * g)=\operatorname{tr}(g * f)$. In 
case where $M$ is symplectic and connected there exists up to normalization a unique such trace [26, 27]. Moreover, this trace functional is of the form

$$
\operatorname{tr}(f)=c \int_{M}\left(f+\sum_{r=1}^{\infty} \lambda^{r} T_{r}(f)\right) \Omega
$$

where $\Omega=\omega \wedge \cdots \wedge \omega$ is the Liouville form, $c \in \mathbb{C}[[\lambda]]$ a normalization factor, and the $T_{r}$ are differential operators. If the star product satisfies $\overline{f * g}=\bar{g} * \bar{f}$, as we assume, then it can easily be shown that by an appropriate choice of the normalization factor $c$ the trace becomes a real functional, i.e. $\operatorname{tr}(\bar{f})=\overline{\operatorname{tr}(f)}$. Since in lowest order of $\lambda$ the trace then consists in integration over $M$ it follows from [12, Lem. 2] that tr becomes a positive functional [10, Lem. 4.3].

Given such a positive trace $\operatorname{tr}$ it is easily seen that supp $\operatorname{tr}=M$ and thus the GNS representation of $\operatorname{tr}$ is faithful according to the last section. Hence $\mathfrak{H}_{\mathrm{tr}} \cong C_{0}^{\infty}(M)[[\lambda]]$ and $\pi_{\mathrm{tr}}$ is equivalent to the left multiplication L. Moreover the Hermitian product is given by $\left\langle\psi_{f}, \psi_{g}\right\rangle=\operatorname{tr}(\bar{f} * g)$. It follows that not only the local commutant is non-trivial as stated in Corollary 4.5 but even the *-local commutant is non-trivial: consider an arbitrary element $f \in C^{\infty}(M)[[\lambda]]$ and the corresponding right multiplication operator $\mathrm{R}_{f}$ on $\mathfrak{H}_{\mathrm{tr}}$. Clearly $\mathrm{R}_{f} \in\left(\pi_{\mathrm{tr}}\left(C^{\infty}(M)[[\lambda]]\right)\right)_{\mathfrak{L}}^{\prime}$ but now we can even prove the existence of $\mathrm{R}_{f}^{*}$ whence $\mathrm{R}_{f} \in\left(\pi_{\mathrm{tr}}\left(C^{\infty}(M)[[\lambda]]\right)\right)_{\mathfrak{L} \mathfrak{B}}^{\prime}$ for every $f \in C^{\infty}(M)[[\lambda]]$. Namely $\mathrm{R}_{f}^{*}$ is given by

$$
\mathrm{R}_{f}^{*}=\mathrm{R}_{\bar{f}},
$$

as an easy computation shows. Thus $\left(\pi_{\mathrm{tr}}\left(C^{\infty}(M)[[\lambda]]\right)\right)_{\mathfrak{L} \mathfrak{B}}^{\prime}$ is non-trivial too.

Another important example is given by the so-called KMS functionals. In [1, 2, 9, 10] the notion of KMS states known from $C^{*}$-algebra theory, see e.g. [13] 15, 20], was transfered to the framework of deformation quantization. We shall only use the final result on the existence and uniqueness of these functionals and their particular form as found in [9, 10]. To this end we first need a notion of star exponential (see e.g. 四), i.e. the analogue of the exponential series build out of star product powers. For our purpose it is sufficient to use the following definition avoiding questions on convergence. The star exponential $\operatorname{Exp}(\beta H) \in C^{\infty}(M)[[\lambda]]$ of $H \in C^{\infty}(M)[[\lambda]]$ with $\beta \in \mathbb{R}$ is defined to be the unique solution in $C^{\infty}(M)[[\lambda]]$ of the differential equation

$$
\frac{d}{d \beta} \operatorname{Exp}(\beta H)=H * \operatorname{Exp}(\beta H)
$$

with initial condition $\operatorname{Exp}(0)=1$. Of course one has to show the existence and uniqueness of such a solution but this has been done e.g. in [10, Lem. 2.2]. Moreover, the usual properties hold, i.e. $\operatorname{Exp}(\beta H)$ commutes with $H$ and satisfies $\operatorname{Exp}\left(\left(\beta+\beta^{\prime}\right) H\right)=\operatorname{Exp}(\beta H) * \operatorname{Exp}\left(\beta^{\prime} H\right)$ as well as $\overline{\operatorname{Exp}(\beta H)}=\operatorname{Exp}(\beta \bar{H})$ for all $\beta, \beta^{\prime} \in \mathbb{R}$ and $H \in C^{\infty}(M)[[\lambda]]$ (due to $\overline{f * g}=\bar{g} * \bar{f}$ ). Note finally, that $\operatorname{Exp}(\beta H)$ can even be extended to arbitrary $\beta \in \mathbb{C}[[\lambda]]$ such that the above relations hold. Then in [10] it was shown that for a given real 'Hamiltonian' $H \in C^{\infty}(M)[[\lambda]]$ and a given 'inverse temperature' $\beta \in \mathbb{R}$ there exists an up to normalization unique KMS functional $\omega_{\mathrm{KMS}}: C_{0}^{\infty}(M)[[\lambda]] \rightarrow \mathbb{C}[[\lambda]]$ given by

$$
\omega_{\mathrm{KMS}}(f)=\operatorname{tr}(\operatorname{Exp}(-\beta H) * f),
$$

where $\operatorname{tr}$ is the trace of $C^{\infty}(M)[[\lambda]]$. We observe that for a positive trace the KMS functional (5.4) is positive too, since $\bar{H}=H$. Moreover, $\operatorname{supp} \omega_{\mathrm{KMS}}=M$ since $\operatorname{Exp}(-\beta H)$ is invertible. Hence again $\mathfrak{H}_{\mathrm{KMS}} \cong C_{0}^{\infty}(M)[[\lambda]]$ as $\mathbb{C}[[\lambda]]$-module and the corresponding GNS representation is again given by left multiplication. Now the Hermitian product is given by

$$
\left\langle\psi_{f}, \psi_{g}\right\rangle_{\mathrm{KMS}}=\operatorname{tr}(\operatorname{Exp}(-\beta H) * \bar{f} * g) .
$$


Again we see that the right multiplication $\mathrm{R}_{f}$ is contained in $\left(\pi_{\mathrm{KMS}}\left(C^{\infty}(M)[[\lambda]]\right)\right)_{\mathfrak{L}}^{\prime}$. A straightforward computation shows that $\mathrm{R}_{f}^{*}$ exists and is given by $\mathrm{R}_{f}^{*}=\mathrm{R}_{\operatorname{Exp}(-\beta H) * \bar{f} * \operatorname{Exp}(\beta H)}$. Thus $\mathrm{R}_{f} \in\left(\pi_{\mathrm{KMS}}\left(C^{\infty}(M)[[\lambda]]\right)\right)_{\mathfrak{L} \mathfrak{B}}^{\prime}$ for all $f \in C^{\infty}(M)[[\lambda]]$ and hence even the *-local commutant of the GNS representation is non-trivial for KMS functionals. This result is expected for physical reasons since KMS functionals are believed to describe thermal behavior and physical situations in thermal equilibrium whence they should be 'mixed'. Note that $\beta=0$ (i.e. infinite temperature) brings us back to the case of the positive trace. We summarize these results in a proposition:

Proposition 5.1 Let $(M, *)$ be a connected symplectic manifold with local star product and positive trace tr. Let $H \in C^{\infty}(M)[[\lambda]]$ be a real Hamiltonian and $\beta \in \mathbb{R}$. Denote by $\omega_{\mathrm{Kms}}$ the corresponding positive KMS functional with GNS pre-Hilbert space $\mathfrak{H}_{\mathrm{KMS}}$. Then one has:

i.) $\operatorname{supp} \omega_{\mathrm{KMS}}=M$ whence $\mathfrak{H}_{\mathrm{KMS}} \cong C_{0}^{\infty}(M)[[\lambda]]$.

ii.) $\mathrm{R}_{f} \in \mathfrak{L} \mathfrak{B}\left(\mathfrak{H}_{\mathrm{KMS}}\right)$ for all $f \in C^{\infty}(M)[[\lambda]]$ and

$$
\mathrm{R}_{f}^{*}=\mathrm{R}_{\operatorname{Exp}(-\beta H) * \bar{f} * \operatorname{Exp}(\beta H)} .
$$

iii.) $\mathrm{R}_{f} \in\left(\pi_{\mathrm{KMS}}\left(C^{\infty}(M)[[\lambda]]\right)\right)_{\mathfrak{L} \mathfrak{B}}^{\prime}$ for all $f \in C^{\infty}(M)[[\lambda]]$ and thus the ${ }^{*}$-local commutant of the GNS representation is non-trivial (if $\operatorname{dim} M>0$ ).

Let us finally investigate the relation between two KMS functionals and their GNS representations. Let $\omega_{\mathrm{KMS}}$ and $\omega_{\mathrm{KMS}}^{\prime}$ be the positive KMS functionals for $(H, \beta)$ and $\left(H^{\prime}, \beta^{\prime}\right)$, respectively, normalized in the same way (5.4). It turns out that the GNS representations are locally and unitarily equivalent by an explicitly given unitary map. Remember that this fact is quite different from the situation in quantum field theory where the GNS representations of KMS states for different temperatures are known to be unitarily inequivalent under quite general pre-conditions, see e.g. [34]. The main point is that the usual representations are type III representations [20] and thus our result suggests that deformation quantization somehow corresponds not to a type III representation. The trace being defined on a twosided ideal $C_{0}^{\infty}(M)[[\lambda]]$ of the whole algebra $C^{\infty}(M)[[\lambda]]$ reminds much more on a type I representation, if heuristically deformation quantization is interpreted as an asymptotic expansion for $\hbar \rightarrow 0$ of some convergent situation. This is not surprising since star products on finite-dimensional symplectic manifolds correspond physically to a finite number of degrees of freedom. If $M$ is even compact, this even looks like a matrix algebra and hence a type $\mathrm{I}_{n}$ with $n \in \mathbb{N}$. Thus a compact symplectic manifold somehow corresponds to a finite dimensional Hilbert space, a result which is also obtained in other quantizations schemes as e.g. geometric quantization [37]. Let us now state the result precisely:

Proposition 5.2 Let $(M, *)$ be a connected symplectic manifold with local star product and positive trace tr. Then for any two real Hamiltonians $H, H^{\prime} \in C^{\infty}(M)[[\lambda]]$ and any two inverse temperatures $\beta, \beta^{\prime} \in \mathbb{R}$ the GNS representations $\pi_{\mathrm{KMS}}$ and $\pi_{\mathrm{KMS}}^{\prime}$ of the corresponding KMS functionals $\omega_{\mathrm{KMS}}$ and $\omega_{\mathrm{KMS}}^{\prime}$ are locally unitarily equivalent via the unitary map $U: \mathfrak{H}_{\mathrm{KMS}} \rightarrow \mathfrak{H}_{\mathrm{KMS}}^{\prime}$ given by

$$
\mathfrak{H}_{\mathrm{KMS}} \ni \psi_{f} \mapsto U \psi_{f}=\psi_{f * \operatorname{Exp}\left(-\frac{\beta}{2} H\right) * \operatorname{Exp}\left(\frac{\beta^{\prime}}{2} H^{\prime}\right)} \in \mathfrak{H}_{\mathrm{KMS}}^{\prime} \cdot
$$

ProOF: Notice that $U$ is indeed well-defined and clearly $U \in \mathfrak{L}\left(\mathfrak{H}_{\mathrm{KMS}}, \mathfrak{H}_{\mathrm{KMS}}^{\prime}\right)$. Then the unitary equivalence is a simple computation. 


\section{Bargmann-Fock representation on Kähler manifolds}

Another fundamental example is given by the formal Bargmann-Fock representation on Kähler manifolds. Before we discuss the general situation let us briefly remember the well-known situation for $M=\mathbb{C}^{n}$. Viewing $\mathbb{C}^{n}$ as Kähler manifold with global holomorphic coordinates $z^{1}, \ldots, z^{n}$ and endowed with the usual symplectic (Kähler) form $\omega=\frac{i}{2} \sum_{k=1}^{n} d z^{k} \wedge d \bar{z}^{k}$ we consider the Wick star product

$$
f *_{\text {Wick }} g=\sum_{r=0}^{\infty} \frac{(2 \lambda)^{r}}{r !} \sum_{i_{1}, \ldots, i_{r}} \frac{\partial^{r} f}{\partial z^{i_{1}} \cdots \partial z^{i_{r}}} \frac{\partial^{r} g}{\partial \bar{z}^{i_{1}} \cdots \partial \bar{z}^{i_{r}}},
$$

where $f, g \in C^{\infty}\left(\mathbb{C}^{n}\right)[[\lambda]]$, see e.g. [11]. It turns out that the evaluation functional $\delta_{p}$ at any point $p \in \mathbb{C}^{n}$ is positive with respect to $*_{\text {wick }}$, and clearly the support of $\delta_{p}$ is given by $\{p\}$. Considering for simplicity the point $p=0$ one finds that the Gel'fand ideal of $\delta_{0}$ is given by [12, Lem. 7]

$$
\mathcal{J}_{0}=\left\{f \in C_{0}^{\infty}\left(\mathbb{C}^{n}\right)[[\lambda]] \mid \forall I: \frac{\partial^{|I|} f}{\partial \bar{z}^{I}}(0)=0\right\}
$$

where $I=\left(\bar{i}_{1}, \ldots, \bar{i}_{r}\right), r \geq 0$, ranges over all multi-indices. Finally, one obtains that the GNS pre-Hilbert space $\mathfrak{H}_{0}$ can be described by $\left(\mathbb{C}\left[\left[\bar{y}^{1}, \ldots, \bar{y}^{n}\right]\right]\right)[[\lambda]]$ where

$$
\mathfrak{H}_{0} \ni \psi_{f} \mapsto \sum_{r=0}^{\infty} \sum_{i_{1}, \ldots, i_{r}} \frac{1}{r !} \frac{\partial^{r} f}{\partial \bar{z}^{i_{1}} \cdots \partial \bar{z}^{i_{r}}}(0) \bar{y}^{i_{1}} \cdots \bar{y}^{i_{r}}
$$

is the isomorphism. Since $\operatorname{supp} \delta_{0}=\{0\}$ is only a single point we see that $\operatorname{supp} \psi_{f}=\{0\}$ for any $\psi_{f} \neq 0$. Thus any $\mathbb{C}[[\lambda]]$-linear endomorphism of $\mathfrak{H}_{0}$ is necessarily local. In order to compute the local commutant of $\pi_{0}\left(C^{\infty}\left(\mathbb{C}^{n}\right)[[\lambda]]\right)$ we recall from [12, Lem. 8] that the GNS representation is given by the formal analogue of the Bargmann-Fock representation

$$
\pi_{0}(f)=\sum_{r, s=0}^{\infty} \frac{(2 \lambda)^{r}}{r ! s !} \sum_{\substack{i_{1}, \ldots, i_{r} \\ j_{1}, \ldots, j_{s}}} \frac{\partial^{r+s} f}{\partial z^{i_{1}} \cdots \partial z^{i_{r}} \partial \bar{z}^{j_{1}} \cdots \partial \bar{z}^{j_{s}}}(0) \bar{y}^{j_{1}} \cdots \bar{y}^{j_{s}} \frac{\partial^{r}}{\partial \bar{y}^{i_{1}} \cdots \bar{y}^{i_{r}}}
$$

where we used the isomorphism $(5.10)$. Then we obtain the following result:

Proposition 5.3 Let $\delta_{0}: C_{0}^{\infty}\left(\mathbb{C}^{n}\right)[[\lambda]] \rightarrow \mathbb{C}[[\lambda]]$ be the evaluation functional at $0 \in \mathbb{C}^{n}$ and $\pi_{0}$ the corresponding GNS representation on $\mathfrak{H}_{0}=\left(\mathbb{C}\left[\left[\bar{y}^{1}, \ldots, \bar{y}^{n}\right]\right]\right)[[\lambda]]$. Then the local and ${ }^{*}$-local commutant of $\pi_{0}\left(C^{\infty}\left(\mathbb{C}^{n}\right)[[\lambda]]\right)$ is trivial.

Proof: We have to show that if an arbitrary $\mathbb{C}[[\lambda]]$-linear endomorphism $L$ of $\mathfrak{H}_{0}$ commutes with all $\pi_{0}(f)$ then it is a multiple of the identity. To this end we can use the additional canonical ring structure of $\mathfrak{H}_{0}$ and in particular the 'vacuum vector' $1 \in \mathfrak{H}_{0}$. Let $L$ be such an endomorphism. Since any left multiplication by elements of $\mathfrak{H}_{0}$ can be realized as $\pi_{0}(f)$ it follows that $L$ commutes with all left multiplications and thus $L$ is itself a left multiplication by the element $L(1) \in \mathfrak{H}_{0}$. On the other hand $L$ commutes with $\pi_{0}\left(z^{i}\right)=\lambda \frac{\partial}{\partial \bar{y}^{i}}$ for all $i$ whence $L(1)$ has to be a constant.

Consider now the general case where $M$ is an arbitrary Kähler manifold with the canonical Fedosov star product $*_{\text {wick }}$ of Wick type as constructed in [11] (see 22, 23] for another approach to such star products of (anti-) Wick type and their classification). In [12, Prop. 9] it was shown that $\delta_{p}$ for any $p \in M$ is a positive $\mathbb{C}[[\lambda]]$-linear functional for this star product. Now again 
$\operatorname{supp} \delta_{p}=\{p\}$ and the whole analysis from above can be repeated completely analogously with the only modification that the Fedosov-Taylor series $\tau_{p}$ at $p$ enters in the analogue of (5.11), see [12, Thm. 5]. But since this map is again surjective (which can be viewed as a sort of quantum Borel lemma [12, Prop. 10]) the same argument as above goes through. We omit here the rather obvious details and state the final result:

Theorem 5.4 Let $\left(M, *_{\text {wick }}\right)$ be a Kähler manifold with canonical Fedosov star product of Wick type and let $p \in M$. Then the local (and ${ }^{*}$-local) commutant of the GNS representation $\pi_{p}\left(C^{\infty}(M)[[\lambda]]\right)$ induced by $\delta_{p}$ is trivial.

\section{Schrödinger representations on cotangent bundles}

Another important class of examples is given by cotangent bundles whose quantization is of particular interest for physics since the typical phase spaces are cotangent bundles of some configuration space manifold. In a series of papers [6] 8, 29, 30] the deformation quantization of cotangent bundles and its relation to pseudo-differential operators and symbol calculus has been extensively discussed and we shall investigate now the locality properties of these star products and their representations. So let us first briefly recall some of the basic results of [6 [8].

One starts with a cotangent bundle $\pi: T^{*} Q \rightarrow Q$ over the so-called configuration space $Q$ which can be embedded as zero section $\iota: Q \hookrightarrow T^{*} Q$ in its cotangent bundle. Given a torsion-free connection $\nabla$ on $Q$ and a positive volume density $\mu \in \Gamma^{\infty}\left(\left|\wedge^{n}\right| T^{*} Q\right)$ on $Q$ one obtains by means of a (slightly modified) Fedosov construction firstly the so-called standard ordered star product $*_{\text {std }}$ which is a homogeneous star product in the sense that the homogeneity operator $\mathrm{H}=\lambda \frac{\partial}{\partial \lambda}+\mathcal{L}_{\xi}$ is a derivation of $*_{\text {Std }}$ where $\mathcal{L}_{\xi}$ is the Lie derivative with respect to the Liouville vector field on $T^{*} Q$. Next we consider the operator [0, Eq. (106)]

$$
N=\exp \left(\frac{\lambda}{2 \mathrm{i}}(\Delta+\mathrm{F}(\alpha))\right)
$$

where $\Delta$ denotes the Laplacian of the semi-Riemannian metric on $T^{*} Q$ induced by the natural pairing of the horizontal and vertical tangent spaces (locally given by $\Delta=\sum_{k} \frac{\partial^{2}}{\partial q^{k} \partial p_{k}}+$ $\sum_{j, k, l} p_{j} \pi^{*} \Gamma_{k l}^{j} \frac{\partial^{2}}{\partial p_{k} \partial p_{l}}+\sum_{j, k} \pi^{*} \Gamma_{j k}^{j} \frac{\partial}{\partial p_{k}}$, where $\Gamma_{k l}^{j}$ are the Christoffel symbols of $\left.\nabla\right)$, and $\mathrm{F}(\alpha)$ is locally given by $\mathrm{F}(\alpha)=\sum_{k} \pi^{*} \alpha_{k} \frac{\partial}{\partial p_{k}}$ where $\alpha=\sum_{k} \alpha_{k} d q^{k}$ is the unique one-form such that $\nabla_{X} \mu=\alpha(X) \mu$ for $X \in \Gamma^{\infty}(T Q)$. Here we have used a canonical (bundle) chart of $T^{*} Q$ but obviously the above expressions are independent of the chart we use, see [6] for a more geometrical description of these operators. Using $N$ as equivalence transformation one defines the Weyl ordered product $*_{\text {weyl }}$ by $f *_{\text {Weyl }} g=N^{-1}\left(N f *_{\text {Std }} N g\right)$, where $f, g \in C^{\infty}\left(T^{*} Q\right)[[\lambda]]$, generalizing thereby the well-known WeylMoyal product from flat $\mathbb{R}^{2 n}$. This star product enjoys the following properties: firstly, we have $\overline{f *_{\text {Weyl }} g}=\bar{g} *_{\text {weyl }} \bar{f}$ and, secondly, the $\mathbb{C}[[\lambda]]$-linear functional

$$
f \mapsto \omega(f)=\int_{Q} \iota^{*} f \mu
$$

defined on $C_{0}^{\infty}\left(T^{*} Q\right)[[\lambda]]$ is positive with respect to $*_{\text {weyl }}$. Note that in $[8]$ this functional was defined on a larger space namely on those functions $f$ with $\iota^{*} f \in C_{0}^{\infty}(Q)[[\lambda]]$ but to be consistent with our notation we shall use $C_{0}^{\infty}\left(T^{*} Q\right)[[\lambda]]$ which turns out to be still 'sufficiently large'. Moreover, the Gel'fand ideal $\mathcal{J}_{\omega}$ is given by those functions $f \in C_{0}^{\infty}\left(T^{*} Q\right)[[\lambda]]$ satisfying $\iota^{*} N f=0$ whence the GNS pre-Hilbert space $\mathfrak{H}_{\omega}$ is isomorphic to the 'formal wave functions' $C_{0}^{\infty}(Q)[[\lambda]]$ on $Q$ by

$$
\mathfrak{H}_{\omega}=C_{0}^{\infty}\left(T^{*} Q\right)[[\lambda]] / \mathcal{J}_{\omega} \ni \psi_{f} \mapsto \iota^{*} N f \in C_{0}^{\infty}(Q)[[\lambda]] .
$$


For technical reasons we choose a smooth cut-off function $\chi: T^{*} Q \rightarrow[0,1]$ such that $\chi$ is equal to 1 in an open neighborhood of $\iota(Q)$ and $\chi \mid T_{q}^{*} Q$ has compact support for each $q \in Q$. Then for $u \in C_{0}^{\infty}(Q)[[\lambda]]$ the map $u \mapsto \psi_{\chi \pi^{*} u}$ is clearly the inverse of the above isomorphism. Note that the usage of $\chi$ is necessary since we have restricted ourselfs to $C_{0}^{\infty}\left(T^{*} Q\right)[[\lambda]]$. Using these isomorphisms one obtains the following explicit formula for the GNS representation $\varrho_{\text {Weyl }}$ of $C^{\infty}\left(T^{*} Q\right)[[\lambda]]$ on $C_{0}^{\infty}(Q)[[\lambda]]$

$$
\varrho_{\mathrm{Weyl}}(f) u=\sum_{r=0}^{\infty} \frac{1}{r !}\left(\frac{\lambda}{\mathrm{i}}\right)^{r} \sum_{i_{1}, \ldots, i_{r}} \iota^{*}\left(\frac{\partial^{r} N f}{\partial p_{i_{1}} \cdots \partial p_{i_{r}}}\right) i_{s}\left(\partial_{q^{i_{1}}}\right) \cdots i_{s}\left(\partial_{q^{i_{r}}}\right) \frac{1}{r !} D^{r} u,
$$

where $i_{s}\left(\partial_{q^{k}}\right)$ denotes the symmetric insertion of the tangent vector $\partial_{q^{k}}$, and $D$ is the operator of symmetric covariant differentiation, locally given by $D=\sum_{k} d q^{k} \vee \nabla_{\partial_{q^{k}}}$, see $\mathbb{8}$, Eq. (7)].

The first trivial observation is that the support of $\omega$ is given by the zero section $\iota(Q)$. Next we want to determine the support of the equivalence class $\psi_{\chi \pi^{*} u}$ and expect that it coincides with $\iota(\operatorname{supp} u)$. This is indeed the case as the following simple verification shows. Let $0 \neq u \in C_{0}^{\infty}(Q)[[\lambda]]$ and consider $\psi_{\chi \pi^{*} u} \in \mathfrak{H}_{\omega}$. Then for $g \in C_{0}^{\infty}\left(T^{*} Q \backslash \iota(\operatorname{supp} u)\right)[[\lambda]]$ we clearly have $\omega_{\chi \pi^{*} u}(g)=0$ whence $\operatorname{supp} \psi_{\chi \pi^{*} u} \subseteq \iota(\operatorname{supp} u)$. The converse inclusion is also true. Let $O \subseteq T^{*} Q$ be open such that $O \cap \iota(\operatorname{supp} u) \neq 0$ then $\pi(O \cap \iota(\operatorname{supp} u))$ is open and non-empty in $Q$. Choose a non-negative function $0 \neq v \in C_{0}^{\infty}(\pi(O \cap \iota(\operatorname{supp} u)))[[\lambda]]$ then clearly $\omega_{\chi \pi^{*} u}\left(\tilde{\chi} \pi^{*} v\right)>0$ where $\tilde{\chi}$ is a suitable smooth bump function equal to 1 in a neighborhood of $O \cap \iota(\operatorname{supp} v)$ such that $\operatorname{supp}\left(\tilde{\chi} \pi^{*} v\right) \subseteq O$. Thus $\iota(\operatorname{supp} u) \subseteq \psi_{\chi \pi^{*} u}$ whence we have shown the following lemma:

Lemma 5.5 Let $\psi_{f} \in \mathfrak{H}_{\omega}$ then $\operatorname{supp} \psi_{f}=\iota\left(\operatorname{supp}\left(\iota^{*} N f\right)\right)$.

Thus the abstract definition of $\operatorname{supp} \psi_{f}$ coincides with the usual geometric support of the corresponding formal wave function on $Q$ (embedded in $T^{*} Q$ ). Hence a local operator on $\mathfrak{H}_{\omega}$ corresponds under the isomorphism (5.14) to a local operator on $C_{0}^{\infty}(Q)[[\lambda]]$ in the usual sense. Thus the general statement of Theorem 3.7 that the GNS representation automatically yields local operators is manifested here by the fact that clearly $\varrho_{\text {Weyl }}(f)$ is a formal series of differential operators and thus local. This observation enables us to compute the local commutant of $\varrho_{\text {Weyl }}\left(C^{\infty}\left(T^{*} Q\right)[[\lambda]]\right)$.

Theorem 5.6 The local commutant $\left(\varrho_{\mathrm{Weyl}}\left(C^{\infty}\left(T^{*} Q\right)[[\lambda]]\right)\right)_{\mathfrak{L}}^{\prime}$ of the Schrödinger representation is trivial if and only if $Q$ is connected. In general $\left(\varrho_{\text {Weyl }}\left(C^{\infty}\left(T^{*} Q\right)[[\lambda]]\right)\right)_{\mathfrak{L}}^{\prime}$ is isomorphic to $H_{0}(Q)[[\lambda]]$.

Proof: Let $A: C_{0}^{\infty}(Q)[[\lambda]] \rightarrow C_{0}^{\infty}(Q)[[\lambda]]$ be a local $\mathbb{C}[[\lambda]]$-linear operator commuting with all $\varrho_{\text {Weyl }}(f)$. Since $A$ is $\mathbb{C}[[\lambda]]$-linear it is of the form $A=\sum_{r=0}^{\infty} \lambda^{r} A_{r}$ and clearly all operators $A_{r}: C_{0}^{\infty}(Q) \rightarrow C_{0}^{\infty}(Q)$ are local again. Since $A$ commutes with all left multiplications by functions $u \in C_{0}^{\infty}(Q)$ the lowest order $A_{0}$ commutes with all such left multiplication. On the other hand by Petree's theorem (see e.g. [24, p. 176]) the locality of $A_{0}$ implies that around any point $q \in Q$ there is a chart such that $A_{0}$ restricted to this chart is a differential operator. Putting this together we see that $A_{0}$ has to be even of order zero in this and hence in any chart, i.e. $A_{0}$ is a left multiplication by a function $a_{0} \in C^{\infty}(Q)$ itself. On the other hand $A_{0}$ commutes with any Lie derivative $\mathcal{L}_{X}$ which can be obtained by $\varrho_{\text {Weyl }}(\hat{X})$ minus some left multiplications where $\hat{X}$ is the function linear in the momentum variables given by $\hat{X}\left(\alpha_{q}\right)=\alpha_{q}\left(X_{q}\right)$ where $\alpha_{q} \in T_{q}^{*} Q$ is a point in $T^{*} Q$ and $X \in \Gamma^{\infty}(T Q)$ is a vector field. Thus $a_{0}$ has to be constant on each connected component. Induction on $r$ completes the proof.

Remark 5.7 i.) In $\left[\right.$ [- 8 ] several generalizations for $*_{\text {Weyl }}$ and $\omega$ have been made: firstly, one can associate to any projectable Lagrangian submanifold $L$ of $T^{*} Q$ a functional $\omega_{L}$ which in the corresponding GNS representation induces the WKB expansion of a Hamiltonian $H$ 
satisfying the Hamilton-Jacobi equation $H \mid L=E$ for some energy value E, see e.g. [3]. In this case the support of the functional $\omega_{L}$ is $L$ and the GNS pre-Hilbert space is isomorphic to $C_{0}^{\infty}(L)[[\lambda]]$ such that the abstract support again corresponds to the geometric support on the Lagrangian sub-manifold. Secondly one can also incorporate a 'magnetic field' as an additional closed two-form on $Q$ pulled back to $T^{*} Q$ and added to the canonical symplectic form. The corresponding star products have GNS representations on a Hermitian line bundle over $Q$ in case where the magnetic field satisfies an additional integrality condition. Then the GNS pre-Hilbert space is isometric to the sections of this line bundle with compact support and the locality structure of the abstract quotient coincides under this isomorphism with the usual notion of the support of the sections. Finally in both cases the local commutant is again trivial if and only if $Q$ is connected. We shall not carry out this in detail since the proof works completely analogously.

ii.) The above examples show that these various kinds of Schrödinger representations as well as the Bargmann-Fock representation with their local commutants indeed behave completely different to the thermal KMS representations. In particular in the case of the Schrödinger representation the connected components of the configuration space $Q$ behave like super selection rules and in fact are the only ones, see also Corollary 3.12.

iii.) Finally this example shows that the notion of irreducible representations does not seem to be appropriate for deformation quantization since clearly $\mathfrak{H}_{\omega}\left(T^{*} O\right) \cong \mathcal{A}_{0}(O)$ is an invariant subspace for all open $O \subseteq Q$. Thus the characterization by local commutants is more suitable. Moreover, the locality structure of the pre-Hilbert space $\mathfrak{H}_{\omega}(Q)$, i.e. the fact that $\mathfrak{H}_{\omega}(O) \perp \mathfrak{H}_{\omega}\left(O^{\prime}\right)$ for $O \cap O^{\prime}=\emptyset$, can be understood as a consequence of the locality structure of the observable algebra. If one heuristically thinks of formal deformation quantization as an 'asymptotic expansion' of some convergent theory, it seems that having a (non-)trivial commutant is a more 'rigid' property of a representation with respect to asymtotic behaviour than being (ir-)reducible.

\section{Strong topologies and von Neumann algebras}

In this section we shall investigate further similarities between local operators in formal GNS representations and bounded operators on complex Hilbert space and end up with certain analogues of von Neumann's double commutant theorem.

Let us first introduce the notion of an approximate identity borrowed from $C^{*}$-algebra theory [14. Def. 2.2.17.]. Let $\left\{O_{n}\right\}_{n \in \mathbb{N}}$ be a sequence of open subsets of $M$ such that each $O_{n}$ has compact closure $O_{n}^{\text {cl }}$ contained in $O_{n+1}$ and such that $M=\bigcup_{n \in \mathbb{N}} O_{n}$. Furthermore let $\chi_{n} \in C_{0}^{\infty}\left(O_{n+1}\right)$ be a smooth function such that $\chi_{n} \mid O_{n}^{\text {cl }}=1$ for all $n \in \mathbb{N}$. Then $\left(O_{n}, \chi_{n}\right)_{n \in \mathbb{N}}$ is called an approximate identity. Note that there always exists such an approximate identity and in the case where $M$ is compact we simply may choose $\chi_{n}=1$ and $O_{n}=M$ for all $n \in \mathbb{N}$.

Now let $(M, *)$ be a Poisson manifold with local star product. Then we consider the space $C_{0}^{\infty}(M)[[\lambda]]$ and its local $\mathbb{C}[[\lambda]]$-linear endomorphisms. Let $\mathcal{A}_{\mathrm{L}}$ and $\mathcal{A}_{\mathrm{R}}$ denote all those endomorphisms obtained by left and right multiplication with elements of $C^{\infty}(M)[[\lambda]]$, respectively. Clearly left and right multiplications commute whence $\mathcal{A}_{\mathrm{L}} \subseteq \mathcal{A}_{\mathrm{R}}^{\prime}$ and $\mathcal{A}_{\mathrm{R}} \subseteq \mathcal{A}_{\mathrm{L}}^{\prime}$, where we may take the commutant in all $\mathbb{C}[[\lambda]]$-linear endomorphisms or in the local ones. Note that canonically $\mathcal{A}_{\mathrm{L}} \cong\left(C^{\infty}(M)[[\lambda]], *\right)$ and $\mathcal{A}_{\mathrm{R}} \cong\left(C^{\infty}(M)[[\lambda]], *\right)^{\mathrm{op}}$, respectively.

Proposition 6.1 Let $(M, *)$ be a Poisson manifold with local star product then

$$
\mathcal{A}_{\mathrm{L}}^{\prime}=\mathcal{A}_{\mathrm{R}} \quad \text { and } \quad \mathcal{A}_{\mathrm{R}}^{\prime}=\mathcal{A}_{\mathrm{L}}
$$


The proof is trivial if $M$ is compact since in this case $1 \in C_{0}^{\infty}(M)[[\lambda]]$ and in the non-compact case one uses an approximate identity.

Though the centers of $\left(C^{\infty}(M)[[\lambda]], *\right), \mathcal{A}_{\mathrm{L}}$, and $\mathcal{A}_{\mathrm{R}}$, respectively, could be rather large in the general Poisson case the centers are known to be trivial in the case where $M$ is a connected symplectic manifold.

Lemma 6.2 Let $(M, *)$ be a connected symplectic manifold with local star product. Then the centers of $\left(C^{\infty}(M)[[\lambda]], *\right), \mathcal{A}_{\mathrm{L}}$, and $\mathcal{A}_{\mathrm{R}}$ are trivial.

In (5.6) we have already noticed that for a KMS functional the adjoints of all right multiplications exits with respect to the induced Hermitian product on $\mathfrak{H}_{\mathrm{KMS}} \cong C_{0}^{\infty}(M)[[\lambda]]$. As a corollary we obtain that both $\mathcal{A}_{\mathrm{L}}$ and $\mathcal{A}_{\mathrm{R}}$, viewed as subalgebras of $\mathfrak{L} \mathfrak{B}\left(\mathfrak{H}_{\mathrm{KMS}}\right)$, are factors if $M$ is connected:

Corollary 6.3 Let $(M, *)$ be a connected symplectic manifold with local star product and let $\omega_{\mathrm{KMS}}$ be a positive KMS functional as in (5.4). Then $\mathcal{A}_{\mathrm{L}}$ and $\mathcal{A}_{\mathrm{R}}$ are factors in $\mathfrak{L} \mathfrak{B}\left(\mathfrak{H}_{\mathrm{KMS}}\right)$.

Since as $\mathbb{C}[[\lambda]]$-modules canonically $\mathfrak{H}_{\omega} \cong C_{0}^{\infty}(M)[[\lambda]]$ (including the corresponding locality structures) when $\omega$ is faithful, we shall now investigate $C_{0}^{\infty}(M)[[\lambda]]$ and the local operators of this space in more detail. In particular we are interested in topological properties of the local operators $\mathfrak{L}\left(C_{0}^{\infty}(M)[[\lambda]]\right)$ and the relation of the topological closures with double commutant closures. To this end we have to specify the topologies we want to use, but firstly it will be necessary to enlarge the framework to more general series. We need (at least) formal Laurent series in $\lambda$, see App. B for definitions. It is clear that all definitions and results are also valid in this setting if we require not only $\mathbb{C}((\lambda))$-linearity but in addition also $\lambda$-adic continuity of all involved maps, as e.g. the positive functional $\omega$, the local operators etc. This is crucial in view of Lemma B.1, and the possible complications cannot be seen in the framework of formal power series since here $\mathbb{C}[[\lambda]]$-linearity implies $\lambda$-adic continuity, see e.g. [17, Prop. 2.1]. Thus let $\omega: C_{0}^{\infty}(M)((\lambda)) \rightarrow \mathbb{C}((\lambda))$ from now on be a $\lambda$-adically continuous, positive and $\mathbb{C}((\lambda))$-linear functional and denote by $\mathfrak{L}\left(\mathfrak{H}_{\omega}\right)$ those $\mathbb{C}((\lambda))$-linear endomorphisms of $\mathfrak{H}_{\omega}$ which are local and $\lambda$-adically continuous. Then it is clear due to Lemma B.1 that $\mathfrak{L}\left(C_{0}^{\infty}(M)((\lambda))\right)$ is given by $\mathfrak{L}\left(C_{0}^{\infty}(M)\right)((\lambda))$ where we denote by $\mathfrak{L}\left(C_{0}^{\infty}(M)\right)$ the (usual) $\mathbb{C}$-linear local operators on $C_{0}^{\infty}(M)$. The $\lambda$-adic topology of $\mathfrak{L}\left(C_{0}^{\infty}(M)\right)((\lambda))$ will be somehow too fine for the study of von Neumann algebras (similar to the norm-topology of bounded operators on complex Hilbert spaces) and thus we are using the following strong operator topology analogously to the usual situation in complex Hilbert spaces. A basis of open neighborhoods of $0 \in \mathfrak{L}\left(C_{0}^{\infty}(M)\right)((\lambda))$ will be given by

$$
O_{f_{1}, \ldots, f_{n} ; \epsilon}:=\left\{A \in \mathfrak{L}\left(C_{0}^{\infty}(M)\right)((\lambda)) \mid \forall l=1, \ldots, k: \varphi\left(A\left(f_{l}\right)\right)<\epsilon\right\},
$$

where $\epsilon>0, f_{1}, \ldots, f_{k} \in C_{0}^{\infty}(M)((\lambda))$, and $\varphi$ is the $\lambda$-adic absolute value. Then a sequence $A_{n}$ of local operators converges strongly, i.e. with respect to this topology, to $A$ if and only if for all $f \in C_{0}^{\infty}(M)((\lambda))$

$$
A_{n} f \rightarrow A f
$$

in the $\lambda$-adic topology of $C_{0}^{\infty}(M)((\lambda))$. Clearly, if $A_{n} \rightarrow A$ in the $\lambda$-adic topology then also $A_{n} \rightarrow A$ in the strong topology. The following example shows that the converse is not true in general:

Example 6.4 Let $M=\mathbb{R}$ and let $\chi_{0}$ be a smooth function having support in $[0,1]$. Define $\chi_{n}(x):=$ $\chi_{0}(x-n)$ for $n \in \mathbb{N}$ and let $A_{n}$ be the left multiplication by $\chi_{n}$. It follows that $A_{n}$ converges strongly to 0 but it does not converge in the $\lambda$-adic topology. 
From the general statement in Prop. B.4 we see that $\mathfrak{L}\left(C_{0}^{\infty}(M)\right)((\lambda))$ is complete with respect to this topology. Hence it makes sense to ask whether a double commutant coincides with a topological closure in order to find at least for particular cases an analogue to von Neumann's double commutant theorem.

We shall now turn again to faithful functionals $\omega$ since in this case $\mathfrak{H}_{\omega} \cong C_{0}^{\infty}(M)((\lambda))$. Thus it will be sufficient to consider the latter space. Moreover, we consider the algebra $\mathcal{A}_{\mathrm{LR}}$ which is generated by all left and right multiplications $\mathcal{A}_{\mathrm{L}}$ and $\mathcal{A}_{\mathrm{R}}$. Note that we have a canonical surjective morphism $\mathcal{A}_{\mathrm{L}} \otimes_{\mathbb{C}((\lambda))} \mathcal{A}_{\mathrm{R}} \rightarrow \mathcal{A}_{\mathrm{LR}}$, simply given by $\mathrm{L}_{f} \otimes \mathrm{R}_{g} \mapsto \mathrm{L}_{f} \mathrm{R}_{g}$ which is not injective since whenever supp $f \cap \operatorname{supp} g=\emptyset$ we have $\mathrm{L}_{f} \mathrm{R}_{g}=0$ but in general $\mathrm{L}_{f} \otimes \mathrm{R}_{g} \neq 0$. Moreover, $\mathcal{A}_{\mathrm{L}}$ and $\mathcal{A}_{\mathrm{R}}$ are canonically embedded in both $\mathcal{A}_{\mathrm{L}} \otimes_{\mathbb{C}((\lambda))} \mathcal{A}_{\mathrm{R}}$ and $\mathcal{A}_{\mathrm{LR}}$.

In the general Poisson case the local commutant of $\mathcal{A}_{\mathrm{LR}}$ can be rather big (since its center can be rather big) but for the connected symplectic case the commutant is trivial due to Lem. 6.2 and Prop. 6.1.

Lemma 6.5 Let $(M, *)$ be a connected symplectic manifold with local star product. Then $\left(\mathcal{A}_{\mathrm{LR}}\right)_{\mathfrak{L}}^{\prime}=$ $\mathbb{C}((\lambda))$ id whence $\left(\mathcal{A}_{\mathrm{LR}}\right)_{\mathfrak{L}}^{\prime \prime}=\mathfrak{L}\left(C_{0}^{\infty}(M)\right)((\lambda))$.

Thus we may now ask whether $\mathcal{A}_{\mathrm{LR}}$ is dense in its double commutant. With the above strong topology this is indeed the case:

Theorem 6.6 Let $(M, *)$ be a connected symplectic manifold with local star product. Then the completion of $\mathcal{A}_{\mathrm{LR}}$ in the strong operator topology is $\mathfrak{L}\left(C_{0}^{\infty}(M)\right)((\lambda))$.

Proof: For a given local operator $L \in \mathfrak{L}\left(C_{0}^{\infty}(M)((\lambda))\right)$ we have to construct a sequence of elements $A_{n} \in \mathcal{A}_{\mathrm{LR}}$ such that $A_{n} \rightarrow L$ strongly. First consider a formal series of local operators $D$ whose coefficients have support in one common compact set contained in some open subset $O$ of $M$. By Peetre's theorem we know, that each coefficient is then a differential operator and by $\mathbb{C}((\lambda))$-linearity we may assume $o(D)=0$ whence we write $D=\sum_{r=0}^{\infty} \lambda^{r} D_{r}$. Consider now $g \in C_{0}^{\infty}(O)$ then $\operatorname{ad}(g)=\mathrm{L}_{g}-\mathrm{R}_{g}$ has order $\geq 1$ and starts in lowest order with the Poisson bracket $\operatorname{ad}(g)=\mathrm{i} \lambda\{g, \cdot\}+\ldots$. Since the Poisson bracket is non-degenerate in the symplectic case, we can obtain by suitable choice of finite algebraic combinations of left multiplications and commutators with elements in $C_{0}^{\infty}(O)$ any differential operator $D_{0}$ up to higher orders in $\lambda$, if we allow for division by finitely many powers of $\lambda$ (actually by $\lambda^{k}$ if the order of differentiation is $k$ ). Thus we obtain $A_{0} \in \mathcal{A}_{\mathrm{LR}}$ having support in $O$ such that $o\left(D-A_{0}\right) \geq 1$ and by induction we find for any $n \in \mathbb{N}$ an element $A_{n} \in \mathcal{A}_{\mathrm{LR}}$ having support in $O$ such that $o\left(D-A_{n}\right) \geq n$ since the higher orders of the operators of left and right multiplications with elements having compact support are differential operators due to the locality of * and Peetre's theorem.

Now let $L$ be a local operator and let $\left(O_{n}, \chi_{n}\right)_{n \in \mathbb{N}}$ be an approximate identity. Then $\chi_{n} L$ is still a local operator having compact support in $O_{n+1}$. Thus we can find a sequence $A_{n} \in \mathcal{A}_{\mathrm{LR}}$ such that $o\left(\chi_{n} L-A_{n}\right) \geq n+1$ for all $n \in \mathbb{N}$. We claim $A_{n} \rightarrow L$ strongly. To prove this let $f \in C_{0}^{\infty}(M)((\lambda))$ where we can assume by $\mathbb{C}((\lambda))$-linearity that $o(f)=0$. Write $f=\sum_{r=0}^{\infty} \lambda^{r} f_{r}$ then for any $k \in \mathbb{N}$ there is a $N \in \mathbb{N}$ such that $N>k$ and $\operatorname{supp} f_{1} \cup \cdots \cup \operatorname{supp} f_{k} \subseteq O_{n}$ for all $n \geq N$. Thus $\chi_{n} L f_{r}=L f_{r}$ for $r=1, \ldots, k$ whence $o\left(\chi_{n} L f-L f\right) \geq k$ for all $n \geq N$. On the other hand $o\left(\chi_{n} L-A_{n}\right) \geq n+1$ uniformly whence also $o\left(\chi_{n} L f-A_{n} f\right) \geq n+1$ since $o(f)=0$ for all $n \geq N$. Then the strong triangle inequality for the order implies that $o\left(L f-A_{n} f\right) \geq k$ for all $n \geq N$ whence indeed $A_{n} \rightarrow L$ in the strong operator topology.

In order to get the full analogy of von Neumann's double commutant theorem we have to take into account the ${ }^{*}$-involution too. Hence we define the ${ }^{*}$-strong operator topology by specifying the following basis of open neighborhoods of 0 :

$$
O_{f_{1}, \ldots, f_{k} ; \epsilon}:=\left\{A \in \mathfrak{L} \mathfrak{B}\left(C_{0}^{\infty}(M)((\lambda))\right) \mid \forall l=1, \ldots, k: \varphi\left(A\left(f_{l}\right)\right)<\epsilon \text { and } \varphi\left(A^{*}\left(f_{l}\right)\right)<\epsilon\right\},
$$


where $\epsilon>0$ and $f_{1}, \ldots, f_{k} \in C_{0}^{\infty}(M)((\lambda)), k \in \mathbb{N}$. Then $A_{n}$ converges * ${ }^{*}$ strongly to $A$ if and only if the sequences $A_{n} f$ and $A_{n}^{*} f$ converge to $A f$ and $A^{*} f$, respectively, in the $\lambda$-adic topology for all $f \in C_{0}^{\infty}(M)((\lambda))$. Note that this topology incorporates now the GNS Hermitian product of the faithful functional $\omega$. Clearly the *-strong operator topology is finer than the strong operator topology whence $A_{n} \rightarrow A^{*}$-strongly implies $A_{n} \rightarrow A$ strongly but the reverse needs not to be true.

Proposition 6.7 Let $(M, *)$ be a Poisson manifold with local star product and $\omega: C_{0}^{\infty}(M)((\lambda)) \rightarrow$ $\mathbb{C}((\lambda))$ be a faithful, positive, $\lambda$-adically continuous, and $\mathbb{C}((\lambda))$-linear functional. Then the space $\mathfrak{L} \mathfrak{B}\left(C_{0}^{\infty}(M)((\lambda))\right)$ is complete in the ${ }^{*}$-strong operator topology.

Proof: Let $A_{n} \in \mathfrak{L} \mathfrak{B}\left(C_{0}^{\infty}(M)((\lambda))\right)$ be a *-strong Cauchy sequence. Since $A_{n} \in \mathfrak{L}\left(C_{0}^{\infty}(M)((\lambda))\right)$ this implies that $A_{n}$ is Cauchy with respect to the strong operator topology, too, and by Prop. B.4 convergent to some $A \in \mathfrak{L}\left(C_{0}^{\infty}(M)((\lambda))\right)$. Similarly $A_{n}^{*}$ converges strongly to some $B \in \mathfrak{L}\left(C_{0}^{\infty}(M)((\lambda))\right)$. Thus it remains to show that $B=A^{*}$ and $A_{n} \rightarrow A^{*}$-strongly. But this is a simple verification using the $\lambda$-adic continuity of $\omega$ and the Hermitian product.

We conclude from Theorem 6.6 and this proposition that for the connected symplectic case with a KMS functional the ${ }^{*}$-strong completion of $\mathcal{A}_{\mathrm{LR}}$ is $\mathfrak{L} \mathfrak{B}\left(C_{0}^{\infty}(M)((\lambda))\right)$ :

Corollary 6.8 Let $(M, *)$ be a connected symplectic manifold and $\omega_{\mathrm{KMS}}$ a positive KMS functional as in (5.4). Then the ${ }^{*}$-strong completion of $\mathcal{A}_{\mathrm{LR}}$ is $\mathfrak{L} \mathfrak{B}\left(C_{0}^{\infty}(M)((\lambda))\right)$.

Note that for the above proofs both the usage of formal Laurent series and the non-degeneracy of the Poisson bracket were crucial. It remains an open and interesting problem whether and how the above theorem can be extended to the general Poisson case. Here a possible degeneracy of the Poisson bracket in certain directions may be compensated by higher orders of the star product. As an example one can consider a symplectic manifold $M$ with star product $*$. Then the substitution $\lambda \mapsto \lambda^{2}$ provides a star product for the Poisson bracket which vanishes identically but clearly the above theorem is still valid in this case. For the general case the above strong operator topology may still be too fine and in order to find a coarser topology one might have to take into account the locally convex topology of $C_{0}^{\infty}(M)$ too.

An analogous theorem is valid for the Schrödinger-like GNS representations on cotangent bundles. With the notation from Section 5 and the extension to formal Laurent series we have $\mathfrak{H}_{\omega} \cong C_{0}^{\infty}(Q)((\lambda))$ and the representation is given by (5.15). Since here (if $Q$ is connected) the local commutant of $\varrho_{\text {Weyl }}\left(C_{0}^{\infty}\left(T^{*} Q\right)((\lambda))\right)$ is already trivial, we expect that the strong closure yields all local operators on $\mathfrak{H}_{\omega}$. This is indeed the case:

Theorem 6.9 Let $Q$ be a connected manifold endowed with a torsion-free connection and a positive density. Then for the corresponding Weyl ordered star product algebra and its Schrödinger representation as in Section 5 the local operators $\mathfrak{L}\left(C_{0}^{\infty}(Q)((\lambda))\right)$ are the completion of $\varrho_{\text {Weyl }}\left(C^{\infty}\left(T^{*} Q\right)((\lambda))\right)$ in the strong operator topology.

Proof: Since we allowed for finitely many negative powers of $\lambda$ we notice from (5.15) that any differential operator on $C_{0}^{\infty}(Q)$ can be expressed as $\varrho_{\text {Weyl }}(f)$ with a suitably chosen $f \in C^{\infty}\left(T^{*} Q\right)((\lambda))$. Thus the claim easily follows from Example B.2 and Prop. B.4.

We conclude this section with a few remarks: Due to the particular and simple form of the Hermitian product in the Schrödinger representation one observes that in this case any local operator has an adjoint. In the general case (even for faithful positive functionals) this is not obvious, whence 
in this case the ${ }^{*}$-strong operator topology is needed. Moreover, if we enlarge the framework to formal CNP series then the strong operator topology can also be written by use of a norm topology of the underlaying Hilbert space over $\mathbb{C}\langle\langle\lambda\rangle\rangle$ since in this case we can define a $\mathbb{C}\langle\langle\lambda\rangle\rangle$-valued norm of $\psi_{f}$ by $\left\|\psi_{f}\right\|:=\sqrt{\left\langle\psi_{f}, \psi_{f}\right\rangle}$. Many aspects of such Hilbert spaces over the field $\mathbb{C}\langle\langle\lambda\rangle\rangle$ were discussed in [12]. One aim to do this could be a 'formal spectral theory' within the local operators in order to compute formal spectra and compare them with asymptotic expansion of their convergent counterparts (if there exists a convergent counterpart).

\section{Tomita-Takesaki theory}

Since the concept of KMS functionals can be formulated for deformation quantization the question for an analogue of the usual Tomita-Takesaki theory arises naturally. It turns out that the deformed algebras allow indeed for such an analogue which will be surprisingly simple: it can be formulated purely algebraically and the usual functional-analytical difficulties do not occur.

Let $(M, *)$ be a connected symplectic manifold and let $H=\sum_{r=0}^{\infty} \lambda^{r} H_{r} \in C^{\infty}(M)[[\lambda]]$ be a real Hamiltonian and $\beta \in \mathbb{R}$ an inverse temperature. Then we denote the corresponding KMS functional by $\omega_{\text {KMS }}$ which is given as in (5.4). The GNS pre-Hilbert space $\mathfrak{H}_{\text {KMS }}$ is then isomorphic to $C_{0}^{\infty}(M)[[\lambda]]$ and in this section we shall always identify them. Using the same notation as for the usual Tomita-Takesaki theory, see e.g. [14, Sect. 2.5.2], we define the $\mathbb{C}[[\lambda]]$-anti-linear operator $S: C_{0}^{\infty}(M)[[\lambda]] \rightarrow C_{0}^{\infty}(M)[[\lambda]]$ by $S f:=\bar{f}$. Since the space $C_{0}^{\infty}(M)[[\lambda]]$ is already complete with respect to the $\lambda$-adic topology $S$ is defined on the whole GNS representation space which drastically simplifies the approach. By a simple computation we see that the operator $F: f \mapsto \operatorname{Exp}(-\beta H) * \bar{f} * \operatorname{Exp}(\beta H)$ is the unique $\mathbb{C}[[\lambda]]$-anti-linear adjoint of $S$, i.e. we have

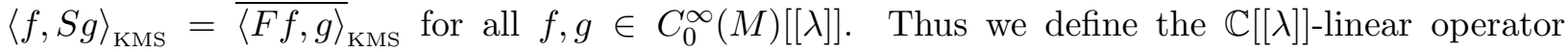
$\Delta:=F S$ as usual and obtain the explicit expression

$$
\Delta f=\operatorname{Exp}(-\beta H) * f * \operatorname{Exp}(\beta H)=\mathrm{L}_{\operatorname{Exp}(-\beta H)} \mathrm{R}_{\operatorname{Exp}(\beta H)} f .
$$

Clearly $\Delta$ is positive with respect to the KMS Hermitian product, i.e. we have $\langle f, \Delta f\rangle \geq 0$ by a simple computation. Moreover, $\Delta$ is obviously invertible with inverse $\Delta^{-1}=\mathrm{L}_{\operatorname{Exp}(\beta H)} \mathrm{R}_{\operatorname{Exp}(-\beta H)}$, and for all $z \in \mathbb{C}[[\lambda]]$ we define $\Delta^{z}:=\mathrm{L}_{\operatorname{Exp}(-z \beta H)} \mathrm{R}_{\operatorname{Exp}(z \beta H)}$ such that $\Delta^{z} \Delta^{z^{\prime}}=\Delta^{z+z^{\prime}}$ and $\Delta^{0}=\mathrm{id}$. Hence we can define the following $\mathbb{C}[[\lambda]]$-anti-linear operator $J: C_{0}^{\infty}(M)[[\lambda]] \rightarrow C_{0}^{\infty}(M)[[\lambda]]$ by

$$
J:=S \Delta^{-\frac{1}{2}} .
$$

A straightforward computation yields the explicit expression

$$
J f=\operatorname{Exp}\left(-\frac{\beta}{2} H\right) * \bar{f} * \operatorname{Exp}\left(\frac{\beta}{2} H\right),
$$

whence in particular $J^{2}=$ id as well as $\langle J f, J g\rangle=\langle g, f\rangle$. Hence $J$ is anti-unitary with $J=J^{*}=$ $J^{-1}$. Finally observe that $J \Delta^{\frac{1}{2}} J=\Delta^{-\frac{1}{2}}$ and $S^{2}=\mathrm{id}=F^{2}$. Analogously to the usual TomitaTakesaki theory we call $J$ the modular conjugation and $\Delta$ the modular operator associated to the KMS functional $\omega_{\mathrm{KMS}}$. It remains to give a reasonable definition of the modular group, i.e. the 'time development' induced by the modular operator. Thus we have to find a reasonable definition for

$\Delta^{\frac{\mathrm{i} t}{\beta \lambda}}$ where the obvious problem comes from the $\lambda$ in the denominator. Note that this is necessary to get the physical dimensions right. Thus a naive definition as for $\Delta^{z}$ is not possible since the star exponential $\operatorname{Exp}\left(\frac{\mathrm{i} t}{\beta \lambda} H\right)$ would not be well-defined in the category of formal power series. Since we do not want to leave this framework we have to give an alternative definition. To motivate 
this, let us proceed heuristically for a moment: if $\Delta^{\frac{\mathrm{i} t}{\beta \lambda}}$ were a one-parameter group of unitaries of $\mathfrak{H}_{\text {KMS }}$ we could try to differentiate it in order to find a differential equation which we can afterwards solve to define the modular group. Since obviously a formal logarithm of $\Delta$ is defined, namely $\ln \Delta=-\beta \operatorname{ad}(H)$, we get the following differential equation (in a strong sense, i.e. after applying to some vector $\left.f \in C_{0}^{\infty}(M)[[\lambda]]\right)$ :

$$
\frac{d}{d t} \Delta^{\frac{\mathrm{i} t}{\beta \lambda}} f=-\frac{\mathrm{i}}{\lambda} \operatorname{ad}(H) \Delta^{\frac{\mathrm{i} t}{\beta \lambda}} f
$$

But this equation now makes perfectly sense since the operator $\operatorname{ad}(H)$ is of order $\lambda$ cancelling the $\lambda$ in the denominator. Moreover, this equation, viewed as an equation for a time-dependent map $U_{t}=\Delta^{\frac{\mathrm{i} t}{\beta \lambda}}$ has indeed a solution, namely the one-parameter group of automorphisms $A_{-t}$ induced by the Heisenberg equation corresponding to the Hamiltonian $H$ provided the flow of the classical Hamiltonian vector field exists for all times $t \in \mathbb{R}$, see e.g. [8, App. B]. Thus we are led to the following definition: Assume in addition that the classical flow of the Hamiltonian vector field of $H_{0}$ exists (in this case the KMS functional is also called a dynamical KMS functional), then we define the modular group by $U_{t}:=A_{-t}$, where $A_{t}$ is the one-parameter group of automorphisms of the quantum time development. Here the minus sign is due to the fact that $f \in C_{0}^{\infty}(M)[[\lambda]]$ is now to be considered as state and not as observable. Using the properties of $A_{t}$ one proves by direct computation that $U_{t}$ is indeed a one-parameter group of unitaries of the GNS pre-Hilbert space $C_{0}^{\infty}(M)[[\lambda]]$, i.e. we have $\left\langle U_{t} f, U_{t} g\right\rangle_{\mathrm{KMS}}=\langle f, g\rangle_{\mathrm{KMS}}$ for all $f, g \in C_{0}^{\infty}(M)[[\lambda]]$ and all $t \in \mathbb{R}$. Together with the fact that $\mathcal{A}_{\mathrm{L}}^{\prime}=\mathcal{A}_{\mathrm{R}}$ (Prop. 6.1) we can now formulate the analogue of the Tomita-Takesaki theorem:

Theorem 7.1 With the notation from above we have:

i.) The $\mathbb{C}[[\lambda]]$-anti-linear map

$$
\mathcal{A}_{\mathrm{L}} \ni \mathrm{L}_{f} \mapsto J \mathrm{~L}_{f} J=\mathrm{R}_{\operatorname{Exp}\left(-\frac{\beta}{2} H\right) * \bar{f} * \operatorname{Exp}\left(-\frac{\beta}{2} H\right)} \in \mathcal{A}_{\mathrm{L}}^{\prime}=\mathcal{A}_{\mathrm{R}}
$$

is a bijection whence $J \mathcal{A}_{\mathrm{L}} J=\mathcal{A}_{\mathrm{L}}^{\prime}$.

ii.) For all $z \in \mathbb{C}[[\lambda]]$ one has

$$
\Delta^{z} \mathcal{A}_{\mathrm{L}} \Delta^{-z}=\mathcal{A}_{\mathrm{L}}
$$

iii.) If in addition the flow of the Hamiltonian vector field of $H_{0}$ exists for all times $t \in \mathbb{R}$, i.e. the $K M S$ functional is a dynamical KMS functional, then one has for all $t \in \mathbb{R}$

$$
U_{t} \mathcal{A}_{\mathrm{L}} U_{-t}=\mathcal{A}_{\mathrm{L}}
$$

Proof: With the above definitions the proof is a simple computation.

This surprisingly simple and algebraic proof (except of the definition of $U_{t}$ ) suggests once more that the algebras of deformation quantization of finite-dimensional symplectic manifolds correspond heuristically to the most simple counterpart in the usual theory of von Neumann algebras, i.e. to the type I. From the physical point of view this can be understood since we have only dealt with finitely many degrees of freedom, whence a type $I_{\infty}$ or even $I_{n}$ is expected for the quantum mechanical description. Hence the full complexity of the usual Tomita-Takesaki theory is not yet reached and hence it would be of major interest to find formulations for infinitely many degrees of 
freedom, where both either a quantum field theoretical or a thermodynamical approach would be very interesting.

On the other hand, the above formulation deals only with the symplectic case. For the general Poisson case many of the above results were not true in general or are rather non-obvious as e.g. the existence or uniqueness of traces. In [35] a classical version for Poisson manifolds is discussed and it would be very interesting to find analogues to these and the above statements for the quantized versions, too.

\section{A Pre-Hilbert spaces over ordered rings and the GNS construc- tion}

For the reader's convenience we shall summarize here some facts on pre-Hilbert spaces over ordered rings and related GNS constructions. See [8, 12] for a detailed exposition and proofs. For the well-known case of $C^{*}$-algebras over complex numbers, see e.g. [13 15, 20] and also [33] for more general complex *-algebras.

First recall that a commutative associative ring $\mathrm{R}$ with $1 \neq 0$ is called ordered with positive elements $\mathrm{P} \subset \mathrm{R}$ if $\mathrm{R}$ is the disjoint union $\mathrm{R}=-\mathrm{P} \dot{\cup}\{0\} \dot{\cup} \mathrm{P}$ and $\mathrm{P}$ is closed under addition and multiplication. If $\mathrm{R}$ is ordered then it is of characteristic zero, i.e. $n 1=1+\cdots+1 \neq 0$ for all $n \in \mathbb{Z}$ and it has no zero divisors. The quotient field $\hat{\mathrm{R}}$ of $\mathrm{R}$ becomes an ordered field such that the usual embedding of $\mathrm{R}$ in $\hat{\mathrm{R}}$ is compatible with the ordering. Now let $\mathrm{R}$ be an ordered ring then we consider $C=R \oplus i R$ where we endow $C$ with a ring structure by requiring $i^{2}=-1$. Then $C$ is again an associative commutative ring with $1 \neq 0$ and has no zero divisors. Elements in $\mathrm{C}$ are written as $z=a+\mathrm{i} b$, where $a, b \in \mathrm{R}$, and $\mathrm{R}$ is embedded in $\mathrm{C}$ via $a \mapsto a+\mathrm{i} 0$. Complex conjugation in $\mathrm{C}$ is defined as usual by $z=a+\mathrm{i} b \mapsto \bar{z}=a-\mathrm{i} b$. Then $z \in \mathrm{C}$ is an element in $\mathrm{R}$ if and only if $z=\bar{z}$ and clearly $\bar{z} z \geq 0$ and $\bar{z} z=0$ if and only if $z=0$.

A pre-Hilbert space over such a ring $\mathrm{C}$ is defined to be a $\mathrm{C}$-module $\mathfrak{H}$ endowed with a $\mathrm{C}$-valued Hermitian product, i.e. a map $\langle\cdot, \cdot\rangle: \mathfrak{H} \times \mathfrak{H} \rightarrow \mathrm{C}$ satisfying the following axioms: $\langle\cdot, \cdot\rangle$ is $\mathrm{C}$-linear in the second argument, $\langle\phi, \psi\rangle=\overline{\langle\psi, \phi\rangle}$ for all $\psi, \phi \in \mathfrak{H}$, and $\langle\cdot, \cdot\rangle$ is positive, i.e. $\langle\psi, \psi\rangle \geq 0$ and $\langle\psi, \psi\rangle=0$ implies $\psi=0$ for all $\psi \in \mathfrak{H}$. Then the Hermitian product satisfies the Cauchy-Schwarz inequality

$$
\langle\phi, \psi\rangle \overline{\langle\phi, \psi\rangle} \leq\langle\phi, \phi\rangle\langle\psi, \psi\rangle, \quad \phi, \psi \in \mathfrak{H} .
$$

If $\mathfrak{H}, \mathfrak{K}$ are pre-Hilbert spaces over $\mathcal{C}$ then a C-linear map $U: \mathfrak{H} \rightarrow \mathfrak{K}$ is called isometric if $\langle U \phi, U \psi\rangle=$ $\langle\phi, \psi\rangle$ for all $\phi, \psi \in \mathfrak{H}$ and unitary if in addition $U$ is surjective. Note that if $U$ is isometric then it is in particular injective, whence a unitary map is invertible and the inverse of a unitary map is unitary again.

Next we consider the possibility to define adjoints of endomorphisms. Let $A: \mathfrak{H} \rightarrow \mathfrak{H}$ be a C-linear endomorphism of a pre-Hilbert space $\mathfrak{H}$ over $C$. Then a $C$-linear endomorphism $B: \mathfrak{H} \rightarrow \mathfrak{H}$ is called adjoint of $A$, written as $B=A^{*}$, if for all $\phi, \psi \in \mathfrak{H}$

$$
\langle B \phi, \psi\rangle=\langle\phi, A \psi\rangle \text {. }
$$

In general the existence of such adjoints is far from being obvious but if $A$ has an adjoint then it is unique. Moreover, if $A^{*}$ and $B^{*}$ exist then $(a A+b B)^{*},(A B)^{*}$, and $\left(A^{*}\right)^{*}$ exist and are given by

$$
(a A+b B)^{*}=\bar{a} A^{*}+\bar{b} B^{*}, \quad(A B)^{*}=B^{*} A^{*}, \quad\left(A^{*}\right)^{*}=A,
$$


where $a, b \in \mathrm{C}$. If $A^{*}$ exists and coincides with $A$ then $A$ is called symmetric. Note that if $U: \mathfrak{H} \rightarrow \mathfrak{H}$ is unitary then $U^{*}$ exists and is given by $U^{-1}$. Finally note that id* $=\mathrm{id}$. Motivated by the familiar case of complex Hilbert spaces one defines

$$
\begin{aligned}
\mathfrak{B}(\mathfrak{H}) & :=\left\{A \in \operatorname{End}(\mathfrak{H}) \mid A^{*} \text { exists }\right\} \\
\mathfrak{U}(\mathfrak{H}) & :=\{U \in \operatorname{End}(\mathfrak{H}) \mid U \text { is unitary }\} .
\end{aligned}
$$

Note that in the particular case where $\mathfrak{H}$ is indeed a Hilbert space over the complex numbers then the Hellinger-Toeplitz theorem ensures that the above definition of $\mathfrak{B}(\mathfrak{H})$ coincides with the bounded operators on $\mathfrak{H}$, see e.g. [31, p. 117]. The following lemma is obvious:

Lemma A.1 Let $\mathfrak{H}$ be a pre-Hilbert space over $\mathrm{C}$ then $\mathfrak{B}(\mathfrak{H})$ is a ${ }^{*}$-algebra with unit element over $\mathrm{C}$ and $\mathfrak{U}(\mathfrak{H}) \subseteq \mathfrak{B}(\mathfrak{H})$ is a group. Moreover, if $\frac{1}{2} \in \mathrm{R}$, then any element in $\mathfrak{B}(\mathfrak{H})$ is a C-linear combination of two symmetric elements.

Now we come to the GNS construction for *-algebras over ordered rings $\mathrm{R}$ and the corresponding quadratic extension $\mathrm{C}$ as above. Let $\mathcal{A}$ be a ${ }^{*}$-algebra over $\mathrm{C}$, i.e. an associative algebra over $\mathrm{C}$ with an $\mathrm{C}$-anti-linear involutive anti-automorphism ${ }^{*}: \mathcal{A} \rightarrow \mathcal{A}$. Then a $\mathrm{C}$-linear functional $\omega: \mathcal{A} \rightarrow \mathrm{C}$ is called positive if

$$
\omega\left(A^{*} A\right) \geq 0
$$

for all $A \in \mathcal{A}$. If $\omega$ is positive then the Cauchy-Schwarz inequality

$$
\begin{aligned}
\omega\left(A^{*} B\right) \overline{\omega\left(A^{*} B\right)} & =\overline{\omega\left(A^{*} B\right)} \leq \omega\left(A^{*} A\right) \omega\left(B^{*} B\right)
\end{aligned}
$$

holds for all $A, B \in \mathcal{A}$ and implies that the space

$$
\mathcal{J}_{\omega}:=\left\{A \in \mathcal{A} \mid \omega\left(A^{*} A\right)=0\right\}
$$

is a left ideal in $\mathcal{A}$, the so-called Gel'fand ideal. The quotient space $\mathfrak{H}_{\omega}:=\mathcal{A} / \mathcal{J}_{\omega}$ thus carries an $\mathcal{A}$-leftmodule structure given by

$$
\pi_{\omega}(A) \psi_{B}:=\psi_{A B},
$$

where $\psi_{B} \in \mathfrak{H}_{\omega}$ denotes the equivalence class of $B$. This representation is called the GNS representation of $\mathcal{A}$ on $\mathfrak{H}_{\omega}$ induced by $\omega$. Moreover $\mathfrak{H}_{\omega}$ becomes a pre-Hilbert space over $\mathrm{C}$ by setting

$$
\left\langle\psi_{A}, \psi_{B}\right\rangle:=\omega\left(A^{*} B\right),
$$

which turns out to be a Hermitian product indeed. Finally $\pi_{\omega}$ is even a ${ }^{*}$-representation, i.e. $\left(\pi_{\omega}(A)\right)^{*}$ always exists for all $A \in \mathcal{A}$ and is given by $\pi_{\omega}\left(A^{*}\right)$. Hence $\pi_{\omega}: \mathcal{A} \rightarrow \mathfrak{B}(\mathfrak{H})$ is a morphism of *-algebras over $\mathrm{C}$.

\section{B Formal series and $\lambda$-adic topology}

In this appendix we shall collect some well-known results on formal series and the $\lambda$-adic topology. The reader is referred to the standard algebra textbooks and for Newton-Puiseux and CNP series we refer to 8 , 12, 32].

In order to define the formal Laurent, Newton-Puiseux, and completed Newton-Puisex (CNP) series we first have to specify the allowed exponents of the formal parameter: Let $S \subset Q$ be a 
subset with either a smallest element $q_{0} \in S$ or $S=\emptyset$. Then $S$ is called CNP-admissable if $S$ has no accumulation point, $N P$-admissable if there exists a $N \in \mathbb{N}$ such that $N \cdot S \subset \mathbb{Z}$, and $L$ admissable if $S \subset \mathbb{Z}$, respectively. Now let $V$ be a module over some ring R and $f: \mathbb{Q} \rightarrow V$ a map. Then one defines the $\lambda$-support of $f$ by $\operatorname{supp}_{\lambda} f:=\{q \in \mathbb{Q} \mid f(q) \neq 0\}$, and the formal Laurent, Newton-Puiseux, and CNP series with coefficients in $V$ by

$$
\begin{gathered}
V((\lambda))=\left\{f: \mathbb{Q} \rightarrow V \mid \operatorname{supp}_{\lambda} f \text { is L-admissable }\right\}, \\
V\left\langle\left\langle\lambda^{*}\right\rangle\right\rangle=\left\{f: \mathbb{Q} \rightarrow V \mid \operatorname{supp}_{\lambda} f \text { is NP-admissable }\right\}, \\
V\langle\langle\lambda\rangle\rangle=\left\{f: \mathbb{Q} \rightarrow V \mid \operatorname{supp}_{\lambda} f \text { is CNP-admissable }\right\},
\end{gathered}
$$

respectively. Observe that $V[[\lambda]] \subseteq V((\lambda)) \subseteq V\left\langle\left\langle\lambda^{*}\right\rangle\right\rangle \subseteq V\langle\langle\lambda\rangle\rangle$ are again R-modules, namely submodules of the R-module of all maps $\mathbb{Q} \rightarrow V$. Elements $f \in V\langle\langle\lambda\rangle\rangle$ are written more familiar as formal series in the formal parameter $\lambda$

$$
f=\sum_{q \in \operatorname{supp}_{\lambda} f} \lambda^{q} f_{q} \quad \text { with } \quad f_{q}=f(q) .
$$

The requirement that $\operatorname{supp}_{\lambda} f$ has in any case a smallest element if $f \neq 0$ is crucial for the definition of the order $o(f):=\min \left(\operatorname{supp}_{\lambda} f\right)$ and one sets $o(0):=+\infty$. One defines the absolute value of $f$ by $\varphi(f):=2^{-o(f)}$ and sets $d_{\varphi}(f, g):=\varphi(f-g)$ for $f, g \in V\langle\langle\lambda\rangle\rangle$, which turns out to define an ultra-metric, following from the strong triangle inequality $o(f+g) \geq \min (o(f), o(g))$ for the order. The induced topology is called the $\lambda$-adic topology and it is well-known that $V[[\lambda]], V((\lambda))$, and $V\langle\langle\lambda\rangle\rangle$ are complete with respect to this metric, whereas $V\left\langle\left\langle\lambda^{*}\right\rangle\right\rangle$ is dense in $V\langle\langle\lambda\rangle\rangle$, see e.g. [12, Prop. 2].

The spaces $\mathrm{R}[[\lambda]], \mathrm{R}((\lambda)), \mathrm{R}\left\langle\left\langle\lambda^{*}\right\rangle\right\rangle$, and $\mathrm{R}\langle\langle\lambda\rangle\rangle$ have a natural ring structure and $V[[\lambda]], V((\lambda))$, $V\left\langle\left\langle\lambda^{*}\right\rangle\right\rangle$, and $V\langle\langle\lambda\rangle\rangle$ become modules over these rings. In case when $\mathrm{R}$ is even a field then $\mathrm{R}((\lambda))$, $\mathrm{R}\left\langle\left\langle\lambda^{*}\right\rangle\right\rangle$, and $\mathrm{R}\langle\langle\lambda\rangle\rangle$ are fields too. We consider now homomorphisms of such modules. If $\phi_{q} \in$ $\operatorname{Hom}_{\mathrm{R}}(V, W)$ is a R-module homomorphisms from $V$ to $W$ for $q \in S \subset \mathbb{Q}$, where $S$ is an L-, NP-, or CNP-admissible set, then $\sum_{q \in S} \lambda^{q} \phi_{q}$ becomes canonically an element of $\operatorname{Hom}_{\mathrm{R}((\lambda))}(V((\lambda)), W((\lambda)))$, $\operatorname{Hom}_{\mathrm{R}\left\langle\left\langle\lambda^{*}\right\rangle\right\rangle}\left(V\left\langle\left\langle\lambda^{*}\right\rangle\right\rangle, W\left\langle\left\langle\lambda^{*}\right\rangle\right\rangle\right)$, or $\operatorname{Hom}_{\mathrm{R}\langle\langle\lambda\rangle\rangle}(V\langle\langle\lambda\rangle\rangle, W\langle\langle\lambda\rangle\rangle)$, respectively, which induces the following inclusions

$$
\begin{gathered}
\operatorname{Hom}_{\mathrm{R}}(V, W)((\lambda)) \subseteq \operatorname{Hom}_{\mathrm{R}((\lambda)))}(V((\lambda)), W((\lambda))) \\
\operatorname{Hom}_{\mathrm{R}}(V, W)\left\langle\left\langle\lambda^{*}\right\rangle\right\rangle \subseteq \operatorname{Hom}_{\mathrm{R}\left\langle\left\langle\lambda^{*}\right\rangle\right\rangle}\left(V\left\langle\left\langle\lambda^{*}\right\rangle\right\rangle, W\left\langle\left\langle\lambda^{*}\right\rangle\right\rangle\right) \\
\operatorname{Hom}_{\mathrm{R}}(V, W)\langle\langle\lambda\rangle\rangle \subseteq \operatorname{Hom}_{\mathrm{R}\langle\langle\lambda\rangle\rangle}(V\langle\langle\lambda\rangle\rangle, W\langle\langle\lambda\rangle\rangle) .
\end{gathered}
$$

In the case of formal power series the corresponding inclusion is known to be indeed an equality, i.e. one has $\operatorname{Hom}_{\mathrm{R}}(V, W)[[\lambda]]=\operatorname{Hom}_{\mathrm{R}[[\lambda]]}(V[[\lambda]], W[[\lambda]])$, see [17, Prop. 2.1], but the above three inclusions are in general proper, see e.g. [8, App. A]. Nevertheless for formal Laurent series one has the following characterization:

Lemma B.1 Let $\Phi: V((\lambda)) \rightarrow W((\lambda))$ be a $\mathrm{R}((\lambda))$-modules homomorphism. Then $\Phi$ is continuous in the $\lambda$-adic topology if and only if $\Phi \in \operatorname{Hom}_{\mathrm{R}}(V, W)((\lambda))$.

Let us remember that if $\mathrm{R}$ is an ordered ring then $\mathrm{R}[[\lambda]], \mathrm{R}((\lambda)), \mathrm{R}\left\langle\left\langle\lambda^{*}\right\rangle\right\rangle$, and $\mathrm{R}\langle\langle\lambda\rangle\rangle$ become ordered rings in a canonical way: let $0 \neq a=\sum_{q \in \operatorname{supp}_{\lambda} a} \lambda^{q} a_{q} \in \mathrm{R}\langle\langle\lambda\rangle\rangle$ then one defines $a>0$ if $a_{\text {min }\left(\operatorname{supp}_{\lambda} a\right)}>0$ in $\mathrm{R}$. It is easily verified that $\mathrm{R}\langle\langle\lambda\rangle\rangle$ is an ordered ring again and $\mathrm{R}[[\lambda]] \subseteq \mathrm{R}((\lambda)) \subseteq$ $\mathrm{R}\left\langle\left\langle\lambda^{*}\right\rangle\right\rangle \subseteq \mathrm{R}\langle\langle\lambda\rangle\rangle$ are ordered sub-rings. Moreover the topology induced by the order coincides with the $\lambda$-adic topology, see e.g. [12, Prop. 3].

We shall now briefly remember the definition of the finite topology of maps and discuss the relation of the $\lambda$-adic topology. Let $V, W$ be $\mathrm{R}$-modules over a ring $\mathrm{R}$ and consider the R-linear 
morphisms $\operatorname{Hom}_{\mathrm{R}}(V, W)$. One defines a topology for $\operatorname{Hom}_{\mathrm{R}}(V, W)$ by specifying a basis of neighborhoods of $0 \in \operatorname{Hom}_{\mathrm{R}}(V, W)$ in the following way: let

$$
O_{v_{1}, \ldots, v_{k}}:=\left\{A \in \operatorname{Hom}_{\mathrm{R}}(V, W) \mid A\left(v_{1}\right)=\cdots=A\left(v_{k}\right)=0\right\},
$$

where $k \in \mathbb{N}$ and $v_{1}, \ldots, v_{k} \in V$. This defines a basis of neighborhoods of 0 and thus (by translating) a topology on $\operatorname{Hom}_{\mathrm{R}}(V, W)$ called the finite topology, see e.g. [21]. As one can easy see it coincides with the compact-open topology of maps when $V$ and $W$ are discretely topologized. Then a sequence $\left(A_{n}\right)_{n \in \mathbb{N}}$ of elements $A_{n} \in \operatorname{Hom}_{\mathrm{R}}(V, W)$ converges to $A \in \operatorname{Hom}_{\mathrm{R}}(V, W)$ if and only if for all $v \in V$ one has $A_{n} v \rightarrow A v$ in the discrete topology of $W$ which is the case if and only if there exists a $N \in \mathbb{N}$ (depending on $v$ ) such that $A_{n} v=A v$ for all $n \geq N$. Cauchy sequences are defined as usual and clearly $\operatorname{Hom}_{\mathrm{R}}(V, W)$ is complete, i.e. any Cauchy sequence converges. The following example shows that the finite topology is quite useful and in general it is strictly coarser than the discrete topology:

Example B.2 Let $M$ be a manifold. Then the completion of the differential operators $\mathfrak{D}\left(C_{0}^{\infty}(M)\right)$ on $C_{0}^{\infty}(M)$ in the finite topology are the local operators $\mathfrak{L}\left(C_{0}^{\infty}(M)\right)$.

Proof: It is straightforward to see that $\mathfrak{L}\left(C_{0}^{\infty}(M)\right)$ is complete in the finite topology and since clearly $\mathfrak{D}\left(C_{0}^{\infty}(M)\right) \subseteq \mathfrak{L}\left(C_{0}^{\infty}(M)\right)$ we only have to construct, for a give $L \in \mathfrak{L}\left(C_{0}^{\infty}(M)\right)$, a sequence $D_{n}$ of differential operators converging to $L$. But this is essentially Peetre's theorem: let $\left(O_{n}, \chi_{n}\right)_{n \in \mathbb{N}}$ be an approximate identity then by Peetre's theorem $D_{n}:=\chi_{n} L$ is a differential operator, since $\chi_{n}$ has compact support. It follows easily that $D_{n} \rightarrow L$ in the finite topology since any $f \in C_{0}^{\infty}(M)$ has support in some $O_{n}$.

Remark B.3 Since the definition of the finite topology as well as the definition of differential operators on an associative, commutative algebra is purely algebraic we note that this example provides a method to define local operators in general, namely as completion of the differential operators in the finite topology. Of course the same can be done for multidifferential operators.

Let us now investigate the connection between the finite topology of $\operatorname{Hom}_{\mathrm{R}}(V, W)$ and the 'strong operator topology' of $\operatorname{Hom}_{\mathrm{R}}(V, W)((\lambda))$ which is defined by the following basis of neighborhoods of 0 : let $\epsilon>0$ and $v_{1}, \ldots, v_{k} \in V$ then we define

$$
O_{v_{1}, \ldots, v_{k}: \epsilon}:=\left\{A \in \operatorname{Hom}_{\mathrm{R}}(V, W)((\lambda)) \mid \forall l=1, \ldots, k: \varphi\left(A\left(v_{l}\right)\right)<\epsilon\right\}
$$

which clearly determines a topology. Here we used the $\lambda$-adic absolute value $\varphi$ on $W$. Clearly a sequence $A_{n} \in \operatorname{Hom}_{\mathrm{R}}(V, W)((\lambda))$ converges to some $A \in \operatorname{Hom}_{\mathrm{R}}(V, W)((\lambda))$ in the strong operator topology if and only if for all $v \in V((\lambda))$ the sequence $A_{n} v$ converges $\lambda$-adically to $A v$ which motivates the name of this topology. Note that the $\lambda$-adic topology of $\operatorname{Hom}_{\mathrm{R}}(V, W)((\lambda))$ is (in general strictly) finer that the strong operator topology, see Example 6.4. The following proposition shows that the finite topology of $\operatorname{Hom}_{\mathrm{R}}(V, W)$ and the strong operator topology of $\operatorname{Hom}_{\mathrm{R}}(V, W)((\lambda))$ fit together very naturally:

Proposition B.4 Let $V, W$ be R-modules and let $\mathfrak{D} \subseteq \mathfrak{L} \subseteq \operatorname{Hom}_{\mathrm{R}}(V, W)$ be subspaces such that $\mathfrak{L}$ is the completion of $\mathfrak{D}$ in the finite topology. Then $\mathfrak{L}((\lambda))$ is the completion of $\mathfrak{D}((\lambda))$ in the strong operator topology of $\operatorname{Hom}_{\mathrm{R}}(V, W)((\lambda))$.

Proof: We shall first show the following lemma which is a particular case of the proposition:

Lemma B.5 $\operatorname{Hom}_{\mathrm{R}}(V, W)((\lambda))$ is complete in the strong operator topology. 
Proof: Let $A_{n} \in \operatorname{Hom}_{\mathrm{R}}(V, W)((\lambda))$ be a Cauchy sequence. Since $W((\lambda))$ is complete in the $\lambda$-adic topology we observe that for any $v \in V((\lambda))$ the sequence $A_{n} v$ is Cauchy and thus convergent in $W((\lambda))$. Thus $A v:=\lim _{n} A_{n} v$ clearly defines a $\mathrm{R}((\lambda))$-linear map $A: V((\lambda)) \rightarrow W((\lambda))$. It thus remains to show that $A$ is $\lambda$-adically continuous and that $A_{n} \rightarrow A$ in the strong operator topology. To this end we write each $A_{n}$ as $A_{n}=\sum_{r=o_{n}}^{\infty} \lambda^{r} A_{n}^{(r)}$ where $o_{n}=o\left(A_{n}\right) \in \mathbb{Z}$ is the order of $A_{n}$. We now assume that the orders $o_{n}$ are not uniformly bounded from below and lead this assumption to a contradiction. We may thus even assume that $o_{n}=-2 n$ by choosing a suitable sub-sequence (the factor 2 is only for technical reasons and we also could have assumed $\left.o_{n}=-2 n-1\right)$. Hence $A_{n}^{(-2 n)} \neq 0$. Choose $v_{0} \in V$ such that $A_{0}^{(0)} v_{0} \neq 0$. Then we can find $v_{1} \in V$ such that $A_{1}\left(v_{0}+\lambda v_{1}\right)$ has a non-vanishing term in order $\lambda^{-1}$ : either $A_{1}^{(-1)} v_{0} \neq 0$ then choose $v_{1}=0$ or $A_{1}^{(-1)} v_{0}=0$ then choose $v_{1}$ such that $A_{1}^{(-2)} v_{1} \neq 0$ which is possible by assumption. Inductively we can find $v_{0}, v_{1}, \ldots, v_{n}, \ldots \in V$ such that $A_{n}\left(v_{0}+\lambda v_{1}+\cdots+\lambda^{n} v_{n}\right)$ has a non-vanishing term in order $\lambda^{-n}$. Defining now $v:=\sum_{n=0}^{\infty} \lambda^{n} v_{n}$ we observe that due to the $\lambda$-adic continuity $A_{n} v$ has a non-vanishing term of order $\lambda^{-n}$ too, whence $-2 n \leq o\left(A_{n} v\right) \leq-n$. But this is in contradiction to $A_{n} v \rightarrow A v$. Thus we conclude that the orders $o_{n}$ are bounded from below by some $N \in \mathbb{Z}$. It clearly follows that $o(A v) \geq N+o(v)$ for all $v \in V((\lambda))$, whence $A$ is $\lambda$-adically continuous. Then $A_{n} \rightarrow A$ in the strong operator topology follows by construction of $A$.

End of the PRoOf of the PRoposition: Now let $L_{n} \in \mathfrak{L}((\lambda))$ be a Cauchy sequence with respect to the strong operator topology. Then $L_{n} \rightarrow L$ with some $L \in \operatorname{Hom}_{\mathrm{R}}(V, W)((\lambda))$ by the lemma. Moreover, we know due to the last lemma that the orders of $L_{n}$ and $L$ are bounded from below by some $N \in \mathbb{Z}$. Considering $v \in V$ we have $L_{n} v \rightarrow L v$ in the $\lambda$-adic topology. In lowest order $N$ this implies that the order $N$ of $L_{n}$ converge to the order $N$ of $L$ in the finite topology of $\operatorname{Hom}_{\mathrm{R}}(V, W)$ whence by Cauchy completeness of $\mathfrak{L}$ we find that the lowest order of $L$ is in $\mathfrak{L}$. Now an easy induction shows that indeed $L \in \mathfrak{L}((\lambda))$ proving the completeness of $\mathfrak{L}((\lambda))$ in the strong operator topology. Consider finally $L \in \mathfrak{L}((\lambda))$ written as $L=\sum_{r=N}^{\infty} \lambda^{r} L^{(r)}$ with $N \in \mathbb{Z}$ and let $D_{n}^{(r)} \in \mathfrak{D}$ be a sequence such that in the finite topology we have $D_{n}^{(r)} \rightarrow L^{(r)}$. We claim that $D_{n}:=\sum_{r=N}^{\infty} \lambda^{r} D_{n}^{(r)} \rightarrow L$ in the strong operator topology which is indeed the case as an easy verification shows. Thus the proposition is shown.

\section{Acknowledgments}

I would like to thank Martin Bordemann for many useful discussions and suggestions, in particular concerning the finite topologies. Moreover, I would like to thank Nikolai Neumaier and Alan Weinstein for valuable discussions. Finally, warm hospitality of the Math Department of UC Berkeley, where this work has been finished, and financial support of the Studienstiftung des deutschen Volkes is gratefully acknowledged.

\section{References}

[1] Basart, H., Flato, M., Lichnerowicz, A., Sternheimer, D.: Deformation Theory applied to Quantization and Statistical Mechanics. Lett. Math. Phys. 8 (1984), 483-394.

[2] Basart, H., Lichnerowicz, A.: Conformal Symplectic Geometry, Deformations, Rigidity and Geometrical (KMS) Conditions. Lett. Math. Phys. 10 (1985), 167-177.

[3] Bates, S., Weinstein, A.: Lectures on the Geometry of Quantization. Berkeley Mathematics Lecture Notes 8, Berkeley, 1995.

[4] Bayen, F., Flato, M., Frønsdal, C., Lichnerowicz, A., Sternheimer, D.: Deformation Theory and Quantization. Ann. Phys. 111 (1978), 61-151.

[5] Bertelson, M., Cahen, M., Gutt, S.: Equivalence of Star Products. Class. Quantum Grav. 14 (1997), A93-A107. 
[6] Bordemann, M., Neumaier, N., Pflaum, M. J., Waldmann, S.: On representations of star product algebras over cotangent spaces on Hermitian line bundles. Preprint Freiburg FR-THEP-98/24 math.QA/9811055 (November 1998).

[7] Bordemann, M., Neumaier, N., Waldmann, S.: Homogeneous Fedosov Star Products on Cotangent Bundles I: Weyl and Standard Ordering with Differential Operator Representation. Commun. Math. Phys. 198 (1998), 363-396.

[8] Bordemann, M., Neumaier, N., Waldmann, S.: Homogeneous Fedosov Star Products on Cotangent Bundles II: GNS Representations, the WKB Expansion, Traces, and Applications. J. Geom. Phys. 29.3 (1999), 199-234.

[9] Bordemann, M., Römer, H., Waldmann, S.: KMS States and Star Product Quantization. Preprint Freiburg FR-THEP-98/17 (August 1998). Contribution to the proceedings of the $30^{\text {th }}$ Symposium on Mathematical Physics inTorun 1998 (Talk by H. Römer). To appear in Rep. Math. Phys.

[10] Bordemann, M., Römer, H., Waldmann, S.: A Remark on Formal KMS States in Deformation Quantization. Lett. Math. Phys. 45 (1998), 49-61.

[11] Bordemann, M., Waldmann, S.: A Fedosov Star Product of Wick Type for Kähler Manifolds. Lett. Math. Phys. 41 (1997), 243-253.

[12] Bordemann, M., Waldmann, S.: Formal GNS Construction and States in Deformation Quantization. Commun. Math. Phys. 195 (1998), 549-583.

[13] Bratteli, O., Robinson, D. W.: Operator Algebras and Quantum Statistical Mechanics II: Equilibrium States. Models in Quantum Statistical Mechanics. Springer-Verlag, New York, Heidelberg, Berlin, 1981.

[14] Bratteli, O., Robinson, D. W.: Operator Algebras and Quantum Statistical Mechanics I: $C^{*}$ and $W^{*}$-Algebras. Symmetry Groups. Decomposition of States. Springer-Verlag, New York, Heidelberg, Berlin, 2. edition, 1987.

[15] Connes, A.: Noncommutative Geometry. Academic Press, San Diego, New York, London, 1994.

[16] DeWilde, M., Lecomte, P. B. A.: Existence of Star-Products and of Formal Deformations of the Poisson Lie Algebra of Arbitrary Symplectic Manifolds. Lett. Math. Phys. 7 (1983), 487-496.

[17] DeWilde, M., Lecomte, P. B. A.: Formal Deformations of the Poisson Lie Algebra of a Symplectic Manifold and Star-Products. Existence, Equivalence, Derivations. In: Hazewinkel, M., GerstenHABER, M. (EDs.): Deformation Theory of Algebras and Structures and Applications, 897-960. Kluwer Academic Press, Dordrecht, 1988.

[18] Fedosov, B. V.: A Simple Geometrical Construction of Deformation Quantization. J. Diff. Geom. 40 (1994), 213-238.

[19] Fedosov, B. V.: Deformation Quantization and Index Theory. Akademie Verlag, Berlin, 1996.

[20] HaAg, R.: Local Quantum Physics. Springer-Verlag, Berlin, Heidelberg, New York, 2. edition, 1993.

[21] Jacobson, N.: Structure of Rings. AMS Coll.Publ. vol. XXXVI, Providence, R.I., 1956.

[22] Karabegov, A. V.: Deformation Quantization with Separation of Variables on a Kähler Manifold. Commun. Math. Phys. 180 (1996), 745-755.

[23] Karabegov, A. V.: Cohomological Classification of Deformation Quantizations with Separation of Variables. Lett. Math. Phys. 43 (1998), 347-357.

[24] Kolář, I., Michor, P. W., Slovák, J.: Natural Operations in Differential Geometry. SpringerVerlag, Berlin, Heidelberg, New York, 1993. 
[25] Kontsevich, M.: Deformation Quantization of Poisson Manifolds, I. Preprint q-alg/9709040 (1997).

[26] Nest, R., Tsygan, B.: Algebraic Index Theorem. Commun. Math. Phys. 172 (1995), 223-262.

[27] Nest, R., Tsygan, B.: Algebraic Index Theorem for Families. Adv. Math. 113 (1995), 151-205.

[28] Omori, H., Maeda, Y., Yoshioka, A.: Weyl Manifolds and Deformation Quantization. Adv. Math. 85 (1991), 224-255.

[29] Pflaum, M. J.: A deformation theoretical approach to Weyl quantization on Riemannian manifolds. Lett. Math. Phys. 45 (1998), 277-294.

[30] Pflaum, M. J.: The normal symbol on Riemannian manifolds. New York J. Math. 4 (1998), 97-125.

[31] Rudin, W.: Functional Analysis. McGraw-Hill Book Company, New York, 2. edition, 1991.

[32] Ruiz, J. M.: The Basic Theory of Power Series. Vieweg Verlag, Braunschweig, Wiesbaden, 1993.

[33] Schmüdgen, K.: Unbounded Operator Algebras and Representation Theory, vol. 37 in Operator Theory: Advances and Applications. Birkhäuser Verlag, Basel, Boston, Berlin, 1990.

[34] Takesaki, M.: Disjointness of the KMS-States of Different Temperatures. Commun. Math. Phys. 17 (1970), 33-41.

[35] Weinstein, A.: The modular automorphism group of a Poisson manifold. J. Geom. Phys. 23 (1997), $379-394$.

[36] Weinstein, A., Xu, P.: Hochschild cohomology and characteristic classes for star-products. Festschrift for V. I. Arnol'd's 60th birthday. Amer. Math. Soc., Providence, 1997.

[37] Woodhouse, N. M. J.: Geometric Quantization. Clarendon Press, Oxford, 1992. 